\title{
SET-THEORETIC SOLUTIONS OF THE YANG-BAXTER EQUATION, BRACES AND SYMMETRIC GROUPS
}

\author{
TATIANA GATEVA-IVANOVA
}

\begin{abstract}
We involve simultaneously the theory of matched pairs of groups and the theory of braces to study set-theoretic solutions of the Yang-Baxter equation (YBE). We show the intimate relation between the notions of a symmetric group (a braided involutive group) and a left brace, and find new results on symmetric groups of finite multipermutation level and the corresponding braces. We introduce a new invariant of a symmetric group $(G, r)$, the derived chain of ideals of $G$, which gives a precise information about the recursive process of retraction of $G$. We prove that every symmetric group $(G, r)$ of finite multipermutation level $m$ is a solvable group of solvable length $\leq m$. To each set-theoretic solution $(X, r)$ of YBE we associate two invariant sequences of symmetric groups: (i) the sequence of its derived symmetric groups; (ii) the sequence of its derived permutation groups and explore these for explicit descriptions of the recursive process of retraction. We find new criteria necessary and sufficient to claim that $(X, r)$ is a multipermutation solution.
\end{abstract}

\section{Contents}

1. Introduction

2. Preliminaries on set-theoretic solutions of YBE 4

3. Matched pairs of groups, symmetric groups, and braces 7

4. The retractions of a symmetric group and its derived chain of ideals. Symmetric groups and symmetric sets of finite multipermutation level

5. The symmetric group $G(X, r)$ of a symmetric set $(X, r)$. Derived symmetric groups and derived permutation groups of a solution

6. A symmetric set $(X, r)$ whose brace $G(X, r)$ is two-sided, or square-free must be a trivial solution

7. Symmetric groups with conditions lri and Raut

Date: July 24, 2018.

2010 Mathematics Subject Classification. Primary 16T25, 81R50, 16W22, Secondary 16S37, 20B35, 81R60, 16W50.

Key words and phrases. Yang-Baxter; Quantum groups; Multipermutation solution; Matched pair of groups; Braided groups; Braces; Permutation groups.

The author was partially supported by Grant I 02/18 "Computational and Combinatorial Methods in Algebra and Applications" of the Bulgarian National Science Fund, by IHÉS, by The Abdus Salam International Centre for Theoretical Physics (ICTP), Trieste, and by Max-Planck Institute for Mathematics, Bonn. 
8. Square-free solutions $(X, r)$ with condition lri on a derived symmetric group, or on a derived permutation group

\section{INTRODUCTION}

Let $V$ be a vector space over a field $k$. It is well-known that the "Yang-Baxter equation" on a linear map $R: V \otimes V \rightarrow V \otimes V$, the equation

$$
R_{12} R_{23} R_{12}=R_{23} R_{12} R_{23}
$$

(where $R_{i, j}$ denotes $R$ acting in the $i, j$ place in $V \otimes V \otimes V$ ), gives rise to a linear representation of the braid group on tensor powers of $V$. When $R^{2}=$ id one says that the solution is involutive, and in this case one has a representation of the symmetric group on tensor powers. A particularly nice class of solutions is provided by set-theoretic solutions, where $X$ is a set and $r: X \times X \rightarrow X \times X$ obeys similar relations on $X \times X \times X$, Dri]. Of course, each such solution extends linearly to $V=k X$ with matrices in this natural basis having only entries from 0,1 and many other nice properties.

Associated to each set-theoretic solution are several algebraic constructions: the monoid $S(X, r)$, the group $G(X, r)$, the semigroup algebra $k S(X, r)=k_{R}[V]$ generated by $X$ with relations $x y=\cdot r(x, y)$ (where - denotes product in the free semigroup, resp free group) and the permutation group $\mathcal{G}(X, r) \subset \operatorname{Sym}(X)$ defined by the corresponding left translations $y \mapsto{ }^{x} y$ for $x \in X$, where $r(x, y)=\left({ }^{x} y, y^{x}\right)$ (under assumptions which will be given later).

$\mathrm{Lu}$, Yan and Zhu, $[\mathrm{LYZ}]$ proposed a general way of constructing set-theoretical solutions of the Yang-Baxter equation using braiding operators on groups, or essentially, the matched pairs of groups. In his survey Takeuchi [Ta gave an introduction to the ESS-LYZ theory, reviewing the main results in [LYZ, ESS, from a matched pair of groups point of view, among them a good way to think about the properties of the group $G(X, r)$ universally generated from $(X, r)$. In particular it is known that the group $G(X, r)$ is itself a braided set in an induced manner and this is the starting point of these works. Following Takeuchi, [Ta, in this paper " a symmetric group" means "a braided group $(G, \sigma)$ with an involutive braiding operator $\sigma$ ". In 2006 Rump introduced new algebraic structures called "braces" as a generalization of radical rings. In a series of works, see for example [Ru06, Ru07, Ru14, he suggested systematic study of braces and developed, "a brace theory approach" to cycle sets and set-theoretic solutions of YBE. Recently the study of braces intensified and the "braces approach" to YBE produced new interesting results, CJO14, BC, BCJ, BCJO, Mat, Ru14, Smok, CGIS16, LV16, AGV], et all.

We propose a new integral approach: to involve simultaneously the theory of matched pairs of groups and the theory of braces in the study of set-theoretic solutions of YBE. In the paper we follow two main directions. 1. We study general symmetric groups and general braces. More specifically, we are interested in 
the properties of symmetric groups $(G, r)$ which have finite multipermutation level, or satisfy various special conditions. 2. We study symmetric sets $(X, r)$ and the associated symmetric groups $G=G(X, r)$ and $\mathcal{G}=\mathcal{G}(X, r)$, each endowed canonically with a left brace structure. In this case we find a strong correlation between certain properties of the generating symmetric set and the properties of $G$ and $\mathcal{G}$, respectively, each of them considered as a symmetric group and as a brace. We find new criteria, each necessary and sufficient to claim that $(X, r)$ is a multipermutation solution (we write $m=\operatorname{mpl} X<\infty$ in this case). The paper is organized as follows. Section 2 contains preliminary material and collects some known results and facts which are used throughout the paper. In Section 3 we discuss matched pairs of groups, symmetric groups in the sense of [Ta] and braces. Theorem 3.6 verifies the equivalence between the notion of a symmetric group $(G, \sigma)$, and the notion of a left brace $(G,+, \cdot)$. It is known that each of these two structures on $G$ implies that $(G, \sigma)$ forms a (non-degenerate) symmetric set in the sense of [ESS, see [LYZ, Ru06]. Sections 4 and [5 are central for the paper. In Section 4 we study the recursive process of retraction of general symmetric groups and braces. Subsect. 4.1 gives some useful technical results, Subsect. 4.2, discusses briefly the retraction of a general symmetric group $(G, r)$. In Subsect. 4.3 we introduce the derived chain of ideals of $G$ (or abbreviated: $D C I$ ), study its properties, and show that it is an invariant of the group which encodes a precise information about the recursive process of retraction on $(G, r)$, see Proposition 4.17 Theorem 4.21 gives some new conditions equivalent to $\operatorname{mpl}(G, r)=m<\infty$. An important application of DCI of a group $(G, r)$ is Theorem 4.22 which proves that every symmetric group $(G, r)$ of finite multipermutation level $m$ is a solvable group of solvable length $\leq m$. In Section 5 we describe the retractions of the symmetric group $\left(G, r_{G}\right)$ associated with a general solution $(X, r)$ and apply these to study the process of retraction of a solution in the most general case. Our starting point is the isomorphism of symmetric groups $\operatorname{Ret}\left(G, r_{G}\right) \simeq\left(\mathcal{G}, r_{\mathcal{G}}\right)$, where $\mathcal{G}=\mathcal{G}(X, r)$ is the corresponding permutation group. To iterate this and describe higher retractions we introduce the notions of derived symmetric groups $\left(G_{j}, r_{G_{j}}\right)=G\left(\operatorname{Ret}^{j}(X, r)\right), j \geq 0$, and derived permutation groups, $\mathcal{G}_{j}=\mathcal{G}\left(\operatorname{Ret}^{j}(X, r)\right), j \geq 0$, related to a solution $(X, r)$. Theorem 5.7 verifies our new formula: $\operatorname{Ret}^{j} G(X, r) \simeq\left(\mathcal{G}\left(\operatorname{Ret}^{j-1}(X, r)\right), j \geq 1\right.$. Theorem 5.15 shows that the symmetric group $G(X, r)$ has finite multipermutation level iff $(X, r)$ is a multipermutation solution. In this case $\operatorname{mpl} \mathcal{G}=m-1 \leq \operatorname{mpl} X \leq \operatorname{mpl} G=m$. Moreover, if $(X, r)$ is a square-free solution of arbitrary cardinality, then $G$ and $X$ have the same multipermutation level, $\operatorname{mpl} G=\operatorname{mpl} X$. In Section 6 we study the brace $(G,+, \cdot)$ canonically associated to a symmetric set $(X, r)$, where $G=G(X, r)$. Theorem 6.1 shows that conditions like " $G$ is a two-sided brace" or " $\left(G, r_{G}\right)$ is square-free" are too rigid, each of them implies that $(X, r)$ is a trivial solution. This motivates our study of some, in general, milder conditions on symmetric groups and their associated left braces. In Sec 7 we introduce special conditions on the actions of a symmetric group $(G, r)$ upon itself, such as lri, or Raut and study the effect of these on the properties of $(G, r)$ and the associated brace $(G,+, \cdot)$. In Section 8 we study square-free solutions $(X, r)$ with condition lri on a derived symmetric group, or on a derived permutation group. Theorem 8.2 shows that the symmetric group $G=G(X, r)$ of a nontrivial square-free solution $(X, r)$ satisfies lri iff $(X, r)$ is a multipermutation solution of level 2 . More generally, $\operatorname{mpl} X=m \geq 2$ iff its derived group $\left(G_{m-2}, r_{G_{m-2}}\right)$ satisfies lri. Theorem 8.5 verifies that $\operatorname{mpl}(X, r)<\infty$, 
whenever $(X, r)$ is a finite square-free solution whose permutation group $\left(\mathcal{G}, r_{\mathcal{G}}\right)$ satisfies lri. The result is generalized by Corollary 8.7 .

\section{Preliminaries on Set-theoretic solutions of YBE}

During the last three decade the study of set-theoretic solutions and related structures notably intensified, a relevant selection of works for the interested reader is WX, GIB, ESS, LYZ, GI04, CES, Ru05, Ta, GIM08, GI11, GI12, GIC12, De15, Mat, CJO14, Ru14, Ve, MS, et all. In this section we recall basic notions and results which will be used in the paper. We shall use the terminology, notation and some results from GI04, GIM07, GIM08, GIM11, GIC12.

Definition 2.1. Let $X$ be a nonempty set (not necessarily finite) and let $r$ : $X \times X \longrightarrow X \times X$ be a bijective map. In this case we use notation $(X, r)$ and refer to it as a quadratic set. The image of $(x, y)$ under $r$ is presented as

$$
r(x, y)=\left({ }^{x} y, x^{y}\right) .
$$

This formula defines a "left action" $\mathcal{L}: X \times X \longrightarrow X$, and a "right action" $\mathcal{R}$ : $X \times X \longrightarrow X$, on $X$ as: $\mathcal{L}_{x}(y)={ }^{x} y, \mathcal{R}_{y}(x)=x^{y}$, for all $x, y \in X$. (i) $r$ is nondegenerate, if the maps $\mathcal{L}_{x}$ and $\mathcal{R}_{x}$ are bijective for each $x \in X$. (ii) $r$ is involutive if $r^{2}=i d_{X \times X}$. (iii) $(X, r)$ is said to be square-free if $r(x, x)=(x, x)$ for all $x \in X$. (iv) $r$ is a set-theoretic solution of the Yang-Baxter equation (YBE) if the braid relation

$$
r^{12} r^{23} r^{12}=r^{23} r^{12} r^{23}
$$

holds in $X \times X \times X$, where $r^{12}=r \times \mathrm{id}_{X}$, and $r^{23}=\mathrm{id}_{X} \times r$. In this case we refer to $(X, r)$ also as a braided set. A braided set $(X, r)$ with $r$ involutive is called $a$ symmetric set.

Remark 2.2. Let $(X, r)$ be a quadratic set. The map $r$ is involutive iff the actions satisfy:

$$
{ }^{u} v\left(u^{v}\right)=u,\left({ }^{u} v\right)^{u^{v}}=v, \forall u, v \in X .
$$

Clearly, an involutive quadratic set $(X, r)$ is square-free iff ${ }^{a} a=a, \forall a \in X$. For a finite symmetric set $(X, r)$ one-sided non-degeneracy implies non-degeneracy.

Convention 2.3. In this paper we shall consider only the case when $r$ is nondegenerate. By "solution" we mean a non-degenerate symmetric set $(X, r)$, where $X$ is a set of arbitrary cardinality. We shall also refer to it as "a symmetric set", keeping the convention that we consider only non-degenerate symmetric sets. It is known that in this case $X$ is embedded in $G(X, r)$. We do not assume (unless stated explicitly) that $(X, r)$ satisfies additional special conditions on the actions.

To each quadratic set $(X, r)$ we associate canonical algebraic objects generated by $X$ and with quadratic defining relations $\Re=\Re(r)$, defined by $x y=z t \in \Re(r)$, iff $r(x, y)=(z, t)$, and $(x, y) \neq(z, t)$. (i) The monoid $S=S(X, r)=\langle X ; \Re\rangle$, with a set of generators $X$ and a set of defining relations $\Re(r)$, is called the monoid associated with $(X, r)$. (ii) The group $G=G(X, r)$ associated with $(X, r)$ is defined as $G=G(X, r)={ }_{g r}\langle X ; \Re\rangle$. (iii) Furthermore, to each non-degenerate braided set $(X, r)$ we also associate a permutation group, denoted $\mathcal{G}=\mathcal{G}(X, r)$, see Definition 
2.5. If $(X, r)$ is a solution, then $S(X, r)$, resp. $G(X, r), \mathcal{G}(X, r)$ is called the YangBaxter monoid, resp. the Yang-Baxter group, resp. the Yang-Baxter permutation group, or shortly the $Y B$ permutation group, associated to $(X, r)$. The YB groups $G(X, r)$ and $\mathcal{G}(X, r)$ will be of particular importance in this paper. In ESS $G(X, r)$ is called the structure group of $(X, r)$, whenever $(X, r)$ is a solution.

Each element $a \in G$ can be presented as a monomial

$$
a=\zeta_{1} \zeta_{2} \cdots \zeta_{n}, \quad \zeta_{i} \in X \bigcup X^{-1} .
$$

We shall consider a reduced form of $a$, that is a presentation (2.2) with minimal length $n$. By convention, length of $a$, denoted by $|a|$ means the length of a reduced form of $a$.

Example 2.4. For arbitrary set $X,|X| \geq 2$, denote by $\tau_{X}=\tau$ the flip map $\tau(x, y)=(y, x)$ for all $x, y \in X$. Then $(X, \tau)$ is a solution called the trivial solution on $X$. Clearly, an involutive quadratic set $(X, r)$ is the trivial solution if and only if ${ }^{x} y=y$, for all $x, y \in X$, or equivalently $\mathcal{L}_{x}=\operatorname{id}_{X}$ for all $x \in X$. In this case $S(X, r)$ is the free abelian monoid generated by $X, G(X, r)$ is the free abelian group, and $\mathcal{G}(X, r)=\left\{\operatorname{id}_{X}\right\}$ is the trivial group.

It is well known that if $(X, r)$ is a braided set, and $G=G(X, r)$, then the equalities

$$
\text { 11: } \quad{ }^{x}\left({ }^{y} z\right)={ }^{x} y\left(x^{y} z\right), \quad \mathbf{r 1}: \quad\left(x^{y}\right)^{z}=\left(x^{y} z\right)^{y^{z}},
$$

hold for all $x, y, z \in X$. So the assignment $x \longrightarrow \mathcal{L}_{x}$ for $x \in X$ extends canonically to a group homomorphism $\mathcal{L}: G \longrightarrow \operatorname{Sym}(X)$, which defines the canonical left action of $G$ on the set $X$. Analogously, there is a canonical right action of $G$ on $X$.

Definition 2.5. Let $(X, r)$ be a non-degenerate braided set, let $\mathcal{L}: G(X, r) \longrightarrow$ $\operatorname{Sym}(X)$ be the canonical group homomorphism defined via the left action. We set $K:=\operatorname{ker} \mathcal{L}$. The image $\mathcal{L}(G(X, r))$ is denoted by $\mathcal{G}=\mathcal{G}(X, r)$ and called the $Y B$-permutation group of $(X, r)$, GI04, GIC12]). We shall often refer to it simply as " $\mathcal{G}(X, r)$ ". The group $\mathcal{G}$ is generated by the set $\left\{\mathcal{L}_{x} \mid x \in X\right\}$.

In a series of works we have shown that the combinatorial properties of a solution $(X, r)$ are closely related to the algebraic properties of its YB structures. Especially interesting are solutions satisfying some of the following conditions.

Definition 2.6. GIM08, GI04 Let $(X, r)$ be a quadratic set.

(1) The following are called cyclic conditions on $X$.

cl1 : $\quad{ }^{\left({ }^{x}\right)} x={ }^{y} x, \quad$ for all $x, y \in X ; \quad$ cr1 : $\quad x^{\left({ }^{x} y\right)}=x^{y}, \quad$ for all $x, y \in X$;

c12: $\left.\quad{ }^{x} y\right) x={ }^{y} x, \quad$ for all $x, y \in X ; \quad$ cr2 : $\quad x^{\left(y^{x}\right)}=x^{y}, \quad$ for all $x, y \in X$.

(2) Condition lri on $(X, r)$ is defined as

$$
\text { lri: } \quad\left({ }^{x} y\right)^{x}=y={ }^{x}\left(y^{x}\right) \text { for all } \quad x, y \in X .
$$

In other words lri holds if and only if $(X, r)$ is non-degenerate, $\mathcal{R}_{x}=\mathcal{L}_{x}^{-1}$, and $\mathcal{L}_{x}=\mathcal{R}_{x}^{-1}$

It is known that every square-free solution $(X, r)$ satisfies the cyclic conditions cc and condition lri, so it is uniquely determined by the left action: $r(x, y)=$ 
$\left(\mathcal{L}_{x}(y), \mathcal{L}_{y}^{-1}(x)\right)$, see [GI04, GIM08]. Solutions with lri are studied in sections 7 and 8.

The cyclic conditions was introduced by the author in GI94, GI96, and was crucial for the proof that every binomial skew polynomial algebra defines canonically (via its relations) a set-theoretic solution of YBE, see GIB. Algebraic objects with relations satisfying the cyclic condition were studied later, GIJO, JO04, CJO06.

Remark 2.7. GIM08, Proposition 2.25. Suppose $(X, r)$ is a quadratic set. Then any two of the following conditions imply the remaining third condition: (i) $(X, r)$ is involutive; (ii) $(X, r)$ is non-degenerate and cyclic; (iii) $(X, r)$ satisfies lri.

Corollary 2.8. Let $(X, r)$ be a solution of arbitrary cardinality. Then $(X, r)$ satisfies condition lri if and only if it satisfies the cyclic conditions $\boldsymbol{c c}$.

The notions of retraction of a symmetric set and multipermutation solutions were introduced in the general case in [ESS]. The active study of multipermutation solutions started in 2004, when the author conjectured that every finite squarefree solution $(X, r)$ has a finite multipermutation level, GI04. Multipermutation solutions were studied in GI04, GIM07, Ru07, GIM08, GIM11, GIC12, CJO10, CJO14, et all.

Let $(X, r)$ be a non-degenerate symmetric set. An equivalence relation $\sim$ is defined on $X$ as $x \sim y$ if and only if $\mathcal{L}_{x}=\mathcal{L}_{y}$. In this case we also have $\mathcal{R}_{x}=\mathcal{R}_{y}$, see ESS. Denote by $[x]$ the equivalence class of $x \in X$ and by $[X]=X / \sim$ the set of equivalence classes. The following can be extracted from GIM08. The left and the right actions of $X$ onto itself naturally induce left and right actions on the retraction $[X]$, via

$$
{ }^{[\alpha]}[x]:=\left[{ }^{\alpha} x\right], \quad[\alpha]^{[x]}:=\left[\alpha^{x}\right], \forall \alpha, x \in X .
$$

The new actions define a canonical map $r_{[X]}:[X] \times[X] \longrightarrow[X] \times[X]$, where $r_{[X]}([x],[y])=\left({ }^{[x]}[y],[x]^{[y]}\right)$. Then $\left([X], r_{[X]}\right)$ is a non-degenerate symmetric set. Note that the canonical map $\mu: X \longrightarrow[X], x \mapsto[x]$ is a braiding-preserving map, (epimorphism of solutions) that is, $(\mu \times \mu) \circ r=r_{[X]} \circ(\mu \times \mu)$. Furthermore, various special properties of $(X, r)$ are inherited by its retraction: (i) $(X, r)$ is lri $\Longrightarrow$ $\left([X], r_{[X]}\right)$ is lri. (ii) $(X, r)$ satisfies the cyclic conditions $\Longrightarrow\left([X], r_{[X]}\right)$ does so. (iii) $(X, r)$ is square-free $\Longrightarrow\left([X], r_{[X]}\right)$ is square-free. The $\operatorname{solution} \operatorname{Ret}(X, r)=$ $\left([X], r_{[X]}\right)$ is called the retraction of $(X, r)$. For all integers $m \geq 0$, $\operatorname{Ret}^{m}(X, r)$ is defined recursively as $\operatorname{Ret}^{0}(X, r)=(X, r), \operatorname{Ret}^{1}(X, r)=\operatorname{Ret}(X, r), \operatorname{Ret}^{m}(X, r)=$ $\operatorname{Ret}\left(\operatorname{Ret}^{m-1}(X, r)\right) .(X, r)$ is a multipermutation solution of level $m$, if $m$ is the minimal number (if any), such that $\operatorname{Ret}^{m}(X, r)$ is the trivial solution on a set of one element. In this case we write $\operatorname{mpl}(X, r)=m$. By definition $(X, r)$ is $a$ multipermutation solution of level 0 if and only if $X$ is a one element set.

The trivial solution $(X, \tau)$ is a multipermutation solution of level 1. More generally, a symmetric set $(X, r)$ has mpl $X=1$ if and only if it is an involutive permutation solution of Lyubashenko, see Example 4.1.

We made the following conjecture in [GI04]: Every finite square-free solution $(X, r)$ is retractable, and therefore it is a multipermutation solution with $\operatorname{mpl}(X, r)<|X|$.

Several independent results verify the Conjecture for the case when the YB-permutation group $\mathcal{G}(X, r)$ is abelian, see [JO10, CJO14, and GIC12, where we give also an 
upper bound for the multipermutation level of $X: \operatorname{mpl} X$ is at most the number of $\mathcal{G}$-orbits of $X$. The Conjecture is also true for all square-free solutions $(X, r)$ of cardinality $\leq 7$. Leandro Vendramin was the first who constructed an infinite family of square free-solutions which are not multipermutation solutions, see $\mathrm{Ve}$, hence the Conjecure is not true for general finite square-free solutions. Moreover Vendramin's counter example of minimal order $(|X|=8)$ satisfies both: (i) $(X, r)$ is an irretractable square-free solution of finite order 8 and (ii) $(X, r)$ is a strong twisted union of two solutions of multipermutation level 2, see for details Remark 5.12. A natural question arises:

Where is the borderline between the classes of retractable and the nonretractable solutions? More generally, what can be said about symmetric sets which are not multipermutation solutions?

Some answers are given by Proposition 4.17, which presents a precise information about the recursive process of retraction on $(G, r)$ in terms of its derived chain of ideals.

It is also natural to search new conditions which imply $\operatorname{mpl}(X, r)<\infty$ and this is one of the main themes discussed in the paper.

\section{MATCHED PAIRS OF GROUPS, SYMMETRIC GROUPS, AND BRACES}

3.1. Matched pairs of groups, braided groups, and symmetric groups. The notion of matched pairs of groups in relation to group factorisation has a classical origin. For finite groups it was used in the 1960's in the construction of certain Hopf algebras KP66. Later such 'bismash product' or 'bicrossed product' Hopf algebras were rediscovered by Takeuchi Ta81 and Majid Ma90, Ma90a, Ma90b. By now there have been many works on matched pairs in different contexts, see Ma95 and references therein. This notion was used by Lu, Yan, and Zhu to study the set-theoretic solutions of YBE and the associated 'braided group', see [LYZ] and the survey [Ta. Matched pairs of monoids were studied in GIM08, and used to characterise general solutions of YBE and extensions of solutions in terms of matched pairs of their associated monoids.

Definition 3.1. Ta, Ma95 A matched pair of groups is a triple $(S, T, \sigma)$, where $S$ and $T$ are groups and $\sigma: T \times S \longrightarrow S \times T, \quad \sigma(a, u)=\left({ }^{a} u, a^{u}\right)$ is a bijective map satisfying the following conditions

$$
\begin{array}{llll}
\text { ML0 : } & { }^{a} 1=1,{ }^{1} u=u, & \text { MR0 : } & 1^{u}=1, a^{1}=a, \\
\text { ML1 : } & { }^{a b} u={ }^{b}\left({ }^{b} u\right), & \text { MR1 : } & a^{u v}=\left(a^{u}\right)^{v}, \\
\text { ML2: } & { }^{a}(u . v)=\left({ }^{a} u\right)\left({ }^{a} v\right), & \text { MR2 : } & (a . b)^{u}=\left(a^{b} u\right)\left(b^{u}\right),
\end{array}
$$

for all $a, b \in T, u, v \in S$.

Definition 3.2. (1) LYZ $A$ braided group is a pair $(G, \sigma)$, where $G$ is a group and $\sigma: G \times G \longrightarrow G \times G$ is a map such that the triple $(G, G, \sigma)$ forms a matched pair of groups, and the left and the right actions induced by $\sigma$ satisfy the compatibility condition:

$$
\text { M3 : } \quad u v=\left({ }^{u} v\right)\left(u^{v}\right) \text { is an equality in } G, \quad \forall u, v \in G \text {. }
$$

(2) Ta A braided group $(G, \sigma)$, with an involutive braiding operator $\sigma$ is called a symmetric group (in the sense of Takeuchi). 
The following results can be extracted from [LYZ, Theorems 1, 2, and 4.

Facts 3.3. (1) The pair $(G, \sigma)$ is a braided group iff conditions $\mathbf{M L 1}, \mathbf{M R} 1$ and the compatibility condition $\boldsymbol{M} 3$ (for $S=T=G$ ) are satisfied. (2) If $(G, \sigma)$ is a braided group, then $\sigma$ satisfies the braid relations and is non-degenerate. So $(G, \sigma)$ forms a non-degenerate braided set in the sense of [ESS. (3) Let $(X, r)$ be a non-degenerate braided set, let $G=G(X, r)$ be the associated $Y B$-group, and let $i: X \longrightarrow G(X, r)$ be the canonical map. Then there is unique braiding operator $r_{G}: G \times G \longrightarrow G \times G$, such that $r_{G} \circ(i \times i)=(i \times i) \circ r_{G}$. In particular, if $X$ is embedded in $G$ then the restriction of $r_{G}$ on $X \times X$ is exactly the map $r$. Furthermore, $\left(r_{G}\right)^{2}=i d_{G \times G}$ iff $r^{2}=i d_{X \times X}$, so $\left(G, r_{G}\right)$ is a symmetric group iff $(X, r)$ is a symmetric set.

We shall refer to the group $\left(G, r_{G}\right)$ as the symmetric group associated to $(X, r)$.

3.2. Braces, definition and basic properties. In 2006 Rump introduced and initiated a systematic study of a new algebraic structure called a brace as a generalization of radical rings. In a series of papers he developed "a brace theory approach" to cycle sets and set-theoretic solutions of YBE, Ru06, Ru07, Ru14, et all. He proved that given a brace $(G,+, \cdot)$ one can define explicitly a left and a right actions on $G$ (considered as a set), which define a non-degenerate involutive set-theoretic solution of YBE denoted by $(G, r)$, see Ru07] and also [CJO14. Rump has shown that for every non-degenerate symmetric set $(X, r)$ one can define canonically an operation + on the YB group $G=G(X, r)$, which makes $(G,+, \cdot)$ a left brace, and therefore a set-theoretic solution of YBE. Recently the number of works on braces increased rapidly and the "braces approach" to YBE produced new interesting results, CJO14, BC, LV16, Mat, Ru14, BCJ, Smok, CGIS16, LV16, AGV, et all. In this paper we work with a definition of a brace, given explicitly in CJO14. It is equivalent to Rump's original definition, Ru06, Ru07.

Definition 3.4. Let $G$ be a set with two operations "." and "+" such that $(G, \cdot)$ is a group, and $(G,+)$ is an abelian group. (i) $(G,+, \cdot)$ is called a left brace if

$$
a(b+c)+a=a b+a c, \quad \text { for all } a, b, c \in G .
$$

(ii) $(G,+, \cdot)$ is a right brace if $(a+b) c+c=a c+b c$, for all $a, b, c \in G$. (iii) $(G,+, \cdot)$ is a two-sided brace if (i) and (ii) are in force. The groups $(G, \cdot)$ and $(G,+)$ are called, respectively, the multiplicative and the additive groups of the brace.

Denote by 0 and $e$, respectively, the neutral elements with respect to the two operations "+" and "." in $G$. Setting $b=0$ in (3.3) one obtains $0=e$ in $G$. In any left brace $(G,+, \cdot)$ one defines another operation $*$ by the rule

$$
a * b=a \cdot b-a-b, \quad \forall a, b \in G .
$$

In general, the operation $*$ in a left brace $G$ is not associative, but it is left distributive with respect to the sum, that is

$$
a *(b+c)=a * b+a * c, \quad \forall a, b, c \in B .
$$

It was proven in $\mathrm{Ru} 07$ that if $(G,+, \cdot)$ is a two-sided brace, then the operation * makes $(G,+, *)$ a Jacobson radical ring. The following facts can be extracted from Rump's works Ru06, Ru07. (1) If $(G,+, \cdot)$ is a left brace, then there is a canonically associated solution $(G, r)$ uniquely determined by the operations in $G$. 
(2) If $G$ is a finite non-trivial two-sided brace, then the (non-degenerate) symmetric set $(G, r)$ associated to $G$ is a multipermutation solution.

We shall prove that, more generally, every symmetric group $(G, r)$ (in the sense of Takeuchi) has a left brace structure, and conversely, on every left brace $G$, one can define a braiding operator $r$, so that $(G, r)$ is a symmetric group, and therefore it is a solution to YBE, see Theorem 3.6 (this is independent of Rump's result).

Suppose $(G,+, \cdot)$ is a set with two operations, such that $(G, \cdot)$ is a group, and $(G,+)$ is an abelian group, but no more restrictions on $G$ are imposed. We define a "left action" $G \times G \longrightarrow G$, on $G$ as:

$$
\mathcal{L}_{a}(b)={ }^{a} b:=a b-a, \quad a, b \in G .
$$

It is straightforward that the map $\mathcal{L}_{a}$ is bijective for each $a \in G$, so we have a map (of sets)

$$
\mathcal{L}: G \longrightarrow \operatorname{Sym}(G), \quad a \mapsto \mathcal{L}_{a}
$$

where $\operatorname{Sym}(G)$ denotes the symmetric group on the set $G$.

We shall verify that $G$ is a left brace iff the map $\mathcal{L}$ defines a left action of the multiplicative group $(G, \cdot)$ upon itself. In this case the equality (3.8) given below presents the operation "+" in terms of the left action and the operation "." The following proposition contains formulae which will be used throughout the paper. The statement extends results of Rump, see also CJO14, Lemma 1. More precisely, the implications $(1) \Longrightarrow(2),(1) \Longrightarrow(3)$ and $(1) \Longrightarrow(4)$ can be extracted from Ru07, see also CJO14. We suggest a direct and independent verification of the equivalence of the four conditions.

Proposition 3.5. Let $(G,+, \cdot)$ be a set with two operations, "." and "+" such that $(G, \cdot)$ is a group and $(G,+)$ is an abelian group. Assume that the map $\mathcal{L}: G \longrightarrow$ $\operatorname{Sym}(G), \quad a \mapsto \mathcal{L}_{a}$, is defined via (3.5). The following conditions are equivalent. (1) $(G,+, \cdot)$ is a left brace, i.e. condition (3.3) holds. (2) G satisfies:

$$
a(b-c)-a=a b-a c, \quad \forall a, b, c \in G .
$$

(3) The map $\mathcal{L}$ determines a left action of the multiplicative group $(G, \cdot)$ on the set $G$, that is

$$
{ }^{(a b)} c={ }^{a}\left({ }^{b} c\right), \quad \forall a, b, c \in G .
$$

(Equivalently, $\mathcal{L}:(G, \cdot) \longrightarrow \operatorname{Sym}(G), a \mapsto \mathcal{L}_{a}$, is a homomorphism of groups). In this case the operation "+" can be presented as

$$
a+b=a\left(a^{-1} b\right), \text { or equivalently, } a+{ }^{a} b=a b, \quad \forall a, b \in G .
$$

(4) For every $a \in G$, the map $\mathcal{L}_{a}$ is an automorphism of the additive group $(G,+)$ that is condition Laut defined below is in force:

$$
\text { Laut : } \quad{ }^{a}(b+c)={ }^{a} b+{ }^{a} c, \quad \forall a, b, c \in G .
$$

In this case the map $\mathcal{L}:(G, \cdot) \longrightarrow \operatorname{Aut}(G,+)$ is a homomorphism of groups.

Proof. (1) $\Longrightarrow(2)$. Assume (1) holds. Let $a, b, c \in G$. We set $d=b-c$, so $b=c+d$, and the left brace condition $a c+a d=a(c+d)+a$, see (3.3), can be written as $a c+a(b-c)-a=a b$ or, equivalently, $a(b-c)-a=a b-a c$, as desired. (2) $\Longrightarrow(1)$. Suppose, $a, c, d \in G$, set $b=d+c$, so $d=b-c$. The equality (3.6) is in force for $a, b, c$, hence replacing $b-c$ with $d$ we obtain $a d-a=a(c+d)-a c$, 
or equivalently $a(c+d)+a=a c+a d$, as desired. (3) $\Longrightarrow(2)$. The equalities ${ }^{a b} c=(a b) c-a b$ and ${ }^{a}\left({ }^{b} c\right)=a(b c-b)-a$ show that $\mathcal{L}$ is a left action (i.e. (3.7) holds) if and only if $(a b) c-a b=a(b c-b)-a$, for all $a, b, c \in G$. Now choose arbitrary $a, b, c \in G$ and set $d=b c$ in the above equality to deduce that it is equivalent to $a d-a b=a(d-b)-a, \quad \forall a, b, d \in G$, which is exactly condition (3.6). (3.7) $\Longrightarrow$ (3.8). Assume (3.7) is in force. We have to show that the operation + and the left action defined via (3.5) are related by the identity (3.8). We set $c={ }^{a^{-1}} b$. Then $a c-a={ }^{a} c={ }^{a}\left(a^{-1} b\right)={ }^{\left(a \cdot a^{-1}\right)} b={ }^{1} b=b$, and therefore $a+b=$ $a c=a\left(a^{-1} b\right)$, as desired. (4) $\Longleftrightarrow(1)$. Suppose $a, b, c \in G$. It follows from the definition of the left action that the equality ${ }^{a}(b+c)={ }^{a} b+{ }^{a} c$ is equivalent to $a(b+c)-a=(a b-a)+(a c-a)$, which, after canceling $-a$ from both sides implies (and is equivalent to) the equality (3.3) defining a left brace.

Note that the left brace $(G,+, \cdot)$ is a two-sided brace iff

$$
\left(a+{ }^{a} b\right) c+c=a c+\left({ }^{a} b\right) c, \quad \forall a, b, c \in G .
$$

Lemma 6.2, (6.1) provides an identity in the multiplicative group $(G, \cdot)$, which is equivalent to (3.10).

\subsection{The close relation between symmetric groups and braces.}

Theorem 3.6. The following two structures on a group $(G, \cdot)$ are equivalent.

(1) The pair $(G, \sigma)$ is a symmetric group, i.e. a braided group with an involutive braiding operator $\sigma$.

(2) $(G,+, \cdot)$ is a left brace.

Furthermore, each of these conditions implies that $(G, \sigma)$ forms a non-degenerate symmetric set, so $(G, \sigma)$ is a solution of $Y B E$.

Proof. (1) $\Longrightarrow(2)$. Define on $G$ a second operation " +" via the formula

$$
a+b:=a\left(\left(^{-1} b\right), \forall a, b \in G .\right.
$$

This is equivalent to $a b=a+{ }^{a} b, \forall a, b \in G$, which is exactly (3.8). We shall prove that the operation + is commutative and associative. By hypothesis $\sigma$ is involutive, it follows then from (2.1) that the actions satisfy:

$$
\left.a^{b}={ }^{(a} b\right)^{-1} a .
$$

The operation + defined above is commutative. This follows from the equalities

$$
\begin{aligned}
a+b & =a\left({ }^{a^{-1}} b\right)=\left({ }^{a}\left(a^{-1} b\right)\right) \cdot\left(a^{\left(^{a^{-1}} b\right)}\right) & & \text { by (3.11) and M3 } \\
& =b \cdot\left(\left(^{\left({ }^{a}\left(a^{-1} b\right)\right)^{-1}} a\right)=b\left(\left(^{b^{-1}} a\right)=b+a\right.\right. & & \text { by (3.12) }
\end{aligned}
$$

Clearly, $a+e=e+a=a$, so the identity element $e$ of the group $G$ is the zeroelement of $(G,+)$. Moreover, $a+\left({ }^{a}\left(a^{-1}\right)\right)=a\left(a^{-1}\right)=e$, for each $a \in G$, so ${ }^{a}\left(a^{-1}\right)=-a$ is the inverse of $a$ in $(G,+)$. We shall verify that the operation + is associative, but before proving this we shall show that the group $G$ acts from the left on $(G,+)$ as automorphisms, that is condition Laut, see (3.9), is in force. Due to the non-degeneracy condition Laut is equivalent to

$$
{ }^{a}\left(b+{ }^{b} c\right)={ }^{a} b+{ }^{a}\left({ }^{b} c\right), \quad \forall a, b, c \in G .
$$


We apply (3.11) and ML2 to the LHS and obtain:

$$
{ }^{a}\left(b+{ }^{b} c\right)={ }^{a}(b c)=\left({ }^{a} b\right)\left({ }^{a^{b}} c\right) .
$$

Compute the RHS applying first ML1 and M3, and then (3.8):

$$
{ }^{a} b+{ }^{a}\left({ }^{b} c\right)={ }^{a} b+{ }^{a} b\left(a^{b} c\right)=\left({ }^{a} b\right)\left(\left(^{b} c\right) .\right.
$$

Now (3.14) and (3.15) imply (3.13), therefore condition Laut is in force. The associativity of the operation + follows from (3.16) and (3.17):

$(3.16)$

$$
\begin{aligned}
& a+(b+c)=a\left(a^{-1}(b+c)\right)=a\left(a^{-1} b+a^{-1} c\right) \quad \text { by (3.11) and Laut } \\
& =a\left(a^{-1} b\right) \cdot\left(\left(^{\left.a^{-1} b\right)^{-1}}\left(a^{-1} c\right)\right)=a\left(a^{-1} b\right)\left(\left(a^{\left(a^{-1}\right.} b\right)\right)^{-1} c\right) \text { by (3.11). } \\
& (a+b)+c=(a+b)\left({ }^{(a+b)^{-1}} c\right)=a\left(^{a^{-1}} b\right)\left(\left(^{\left.\left(a^{(-1} b\right)\right)^{-1}} c\right) .\right.
\end{aligned}
$$

We have verified that $(G,+)$ is an abelian group, and the multiplicative group $(G, \cdot)$ acts from the left on $(G,+)$ as automorphisms. By (3.8) the left action can be presented as ${ }^{a} b=a b-a, \forall a, b \in G$. In other words one has ${ }^{a} b=\mathcal{L}_{a}(b)$, for all $a, b \in G$, where the maps $\mathcal{L}_{a}$ are defined in (3.5). This implies that $(G,+, \cdot)$ satisfies the hypothesis of Proposition 3.5. and condition Laut is in force, hence $G$ is a left brace, see Prop. 3.5 (4). The implication (1) $\Longrightarrow(2)$ has been proved.

$(2) \Longrightarrow(1)$. Some steps in this implication can be extracted from CJO14. For completeness (and simplicity) we give a different proof which is compatible with our settings and involves relations and conditions that will be used throughout the paper. Assume $(G,+, \cdot)$ is a left brace. We first define a left and a right action of $(G, \cdot)$ upon itself. As in the previous subsection define the bijective maps $\mathcal{L}_{a}: G \times G \longrightarrow G$ as $\mathcal{L}_{a}(b)={ }^{a} b:=a b-a$, see (3.5). It follows from Proposition 3.5 that the map

$$
\mathcal{L}: G \longrightarrow \operatorname{Sym}(G) \quad a \mapsto \mathcal{L}_{a}
$$

defines a left action of $(G, \cdot)$ upon itself, that is condition ML1 and ML0 hold. Furthermore, condition Laut and the equalities (3.11), (3.8) are in force. We next define a right "action" of $(G, \cdot)$ upon itself, $\mathcal{R}: G \longrightarrow \operatorname{Sym}(X), b \mapsto \mathcal{R}_{b}$, by the equality

$$
\mathcal{R}_{b}(a)=a^{b}:={ }^{\left({ }^{a} b\right)^{-1}} a, \quad \forall a, b \in G .
$$

We shall verify that this is, indeed, an action. Clearly, MR0 is in force, we have to verify MR1. The argument will involve the compatibility condition M3, see (3.2) and condition ML2, so we shall prove these first. Condition M3 is verified by the following equalities:

$$
\begin{aligned}
a b=a+{ }^{a} b & ={ }^{a} b+a & & \text { by (3.8), and " }+ \text { commutative" } \\
& =\left({ }^{a} b\right)\left(\left(^{a} b\right)^{-1} a\right)=\left({ }^{a} b\right)\left(a^{b}\right) & & \text { by (3.11) }
\end{aligned}
$$

Next we show that ML2 is also in force. Indeed, one has

$$
\begin{aligned}
{ }^{a}(b c) & ={ }^{a}\left(b+{ }^{b} c\right)={ }^{a} b+{ }^{a}\left({ }^{b} c\right) & & \text { by (3.8) }) \text { and Laut } \\
& ={ }^{a} b+{ }^{\left({ }^{a} b . a^{b}\right)} c=\left({ }^{a} b\right)\left({ }^{\left(a^{b}\right)} c\right) & & \text { by ML1, M3, and (3.8). }
\end{aligned}
$$

Finally we verify MR1. Let $a, b, c \in G$. One has

$$
\begin{aligned}
& a^{(b c)}={ }^{\left({ }^{a}(b c)\right)^{-1}} a=\left(\left(^{a} b\right)\left(a^{b} c\right)\right)^{-1} a \quad \text { by (3.18) and by ML2 }
\end{aligned}
$$

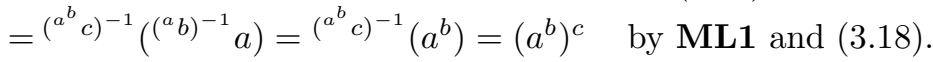


Hence condition MR1 is in force, and therefore the formula (3.18) defines a right action of $(G, \cdot)$ upon itself.

We have shown that the (multiplicative) group $(G, \cdot)$ acts upon itself from the left and from the right via the actions defined above. These actions satisfy ML1, MR1, ML2 and the compatibility condition M3. Define a map

$$
\sigma: G \times G \longrightarrow G \times G, \quad \sigma(a, b):=\left({ }^{a} b, a^{b}\right) .
$$

It follows from the results of [LYZ, see Fact 3.3, that the pair $(G, \sigma)$ is a braided group, so condition MR2 is also in force. To show that the map $\sigma$ is involutive we verify conditions (2.1). The definition (3.18) of the right action implies straightforwardly that $\left.{ }^{a} b\left(a^{b}\right)={ }^{a} b\left({ }^{(a} b\right)^{-1} a\right)=a$, for all $a, b \in G$. We leave the reader to verify the remaining equality $\left({ }^{a} b\right)^{a^{b}}=b$, for all $a, b \in G$. We have shown that $(G, \sigma)$ is a symmetric group (in the sense of Takeuchi), so the implication $(2) \Longrightarrow(1)$ is in force.

It follows from Fact 3.3 again that each of the equivalent conditions (1) and (2) implies that $(G, \sigma)$ forms a non-degenerate symmetric set in the sense of [ESS].

Till the end of the paper we shall assume that the following is in force.

Definition-Convention 3.7. (1) Let $(G, \sigma)$ be a symmetric group. We shall always consider the left and the right actions of $G$ upon itself defined via the formula $\sigma(u, v)=\left({ }^{u} v, u^{v}\right)$. We call the left brace $(G,+, \cdot)$ with operation "+" defined via (3.11) the left brace associated to $(G, \sigma)$.

(2) Conversely, given a left brace $(G,+, \cdot)$, the associated symmetric group $(G, \sigma)$ is "built" on the multiplicative group of the brace $(G, \cdot)$ with a left action $\mathcal{L}$ and a right action $\mathcal{R}$ defined as in the proof of Theorem 3.6, see (3.5) and (3.18), respectively.

(3) Let $(X, r)$ be a (non-degenerate) symmetric set with YB-group $G=G(X, r)$, let $\left(G, r_{G}\right)$ be the symmetric group associated to $(X, r)$, see Facts 3.3 . The left brace $(G,+, \cdot)$ associated to $\left(G, r_{G}\right)$ is called the left brace associated to the solution $(X, r)$.

Theorem 3.6 gives a valuable information on left braces: every left brace $(G,+, \cdot)$ is equipped (canonically) with an involutive braiding operator $r_{G}$, so that $G$ has an additional structure - the structure of a symmetric group. In particular, the powerful matched pairs conditions ML2 and MR2 in $G$ can be and will be used to study various properties of the given brace. We shall study the close relation between the properties of a symmetric set $(X, r)$ and the corresponding two structures (i) the symmetric group $\left(G, r_{G}\right)$, and (ii) the left brace $(G,+, \cdot)$ each of them built on the YB group $G=G(X, r)$. Theorem 3.6 can be used also to directly "translate" results from braces to braided groups or vice versa. The theorem was already applied in this way by Agata Smoktunowicz, who obtained interesting results on two-sided braces and Engel groups, see [Smok.

3.4. More details about the YB monoid $S=S(X, r)$. Let $(X, r)$ be a solution, let $S=S(X, r)$ be its associated monoid, and let $\left(G, r_{G}\right),(G,+, \cdot)$ be its associated symmetric group and left brace, respectively. We shall prove, see Proposition 3.11. that if the monoid $S=S(X, r)$ is embedded in $G=G(X, r)$ then $S$ is invariant under the actions of $G$ upon itself. 
Remark 3.8. Recall that a subset $Y \subset X$ is $r$-invariant if $r(Y \times Y) \subseteq Y \times Y$. The restricted map $r_{Y}=\left(r_{\mid Y \times Y}\right): Y \times Y \longrightarrow Y \times Y$ defines canonically the induced solution $\left(Y, r_{Y}\right)$ on $Y$. A nonempty subset $Y \subset X$ is a (left) $G$-invariant subset of $X$, if $Y$ is invariant under the left action of $G$. Since $r$ is involutive, $Y$ is left $G$-invariant if and only if it is right $G$-invariant, so we shall refer to it simply as $a G$ invariant subset. Each $G$-invariant subset $Y$ of $X$ is also $r$-invariant, the converse, in general, is not true. Clearly, $Y$ is (left) $G$-invariant iff it is a $\mathcal{G}$-invariant subset. Each $G$-orbit $X_{0}$ under the left action of $G$ on $X$ is $G$-invariant and therefore it is an $r$-invariant subset.

Remark 3.9. It follows from [ESS, and [LY], p.18, that the additive group $(G,+)$ is isomorphic to the free abelian group, ${ }_{g r}[X]$ generated by $X$, so the set $X$ is embedded in $G$. It is well-known that if $(X, r)$ is a finite symmetric set then the monoid $S=S(X, r)$ is with cancellation law and satisfies the Öre conditions. The YB group $G$ is the group of quotients of $S$, so $S$ is embedded in $G$ (clearly $X$ is embedded in $S$ ).

Convention 3.10. In the cases when $(X, r)$ is an infinite symmetric set we shall assume that the associated YB monoid $S=S(X, r)$ is embedded in $G=G(X, r)$.

Note that for any pair $x, y \in X \times X$ the only nontrivial equality of degree 2 in the monoid $S$ involving the word $x y$ (if any) has the shape $x y={ }^{x} y \cdot x^{y}$.

Proposition 3.11. Let $(X, r)$ be a non-degenerate symmetric set, $S=S(X, r), G=$ $G(X, r),\left(G, r_{G}\right)$ in the usual notation. Then the following three conditions hold.

(1) The monoid $S$ is invariant under the left and the right actions of $G$ upon itself, so $S$ is an $r_{G}$-invariant subset of $\left(G, r_{G}\right)$.

(2) Let $r_{S}: S \times S \longrightarrow S \times S$ be the restriction of $r_{G}$ on $S \times S$. Then $\left(S, r_{S}\right)$ is a solution. Moreover, it is a braided M3-monoid in the sense of GIM08.

(3) $S$ is closed under the operation + in $G$. So $(S,+, \cdot)$ is an algebraic structure such that $(i)(S, \cdot)$ is a monoid with unit 1. (ii) $(S,+)$ is an abelian monoid generated by $X$ and with the same neutral element $(0=1)$. More precisely, $(S,+)$ is isomorphic to the free abelian monoid mon $[X]$ generated by $X$. (iii) the left brace equality $a(b+c)+a=a b+a c$ holds for all $a, b, c \in S$;

Proof. (1). One has ${ }^{a} 1=1, \forall a \in G$ (by ML0). We use induction on the length $|u|$ of $u \in S$ to show that ${ }^{a} u \in S$ for all $a \in G, u \in S$. The group $G$ acts on $X$ from the left, so this gives the base for the induction. Assume ${ }^{a} u \in S$ for all $a \in G$ and all, $u \in S$ with $|u| \leq n$. Suppose $a \in G, u \in S$ with $|u|=n+1$. Then $u=v x$, where $x \in X, v \in S,|v|=n$. We use ML2 to yield ${ }^{a} u={ }^{a}(v x)=\left({ }^{a} v\right)\left({ }^{\left(a^{v}\right)} x\right)$. One has ${ }^{\left(a^{v}\right)} x \in X$, and by the inductive assumption ${ }^{a} v \in S$, hence ${ }^{a} u \in S, \forall a \in G, u \in S$. It follows that $S$ is invariant under the left action of $G$ upon itself. The braiding operator $r_{G}$ is involutive, hence $S$ is also invariant under the right action of $G$ upon itself. Therefore $S$ is $r_{G}$-invariant. (2). The left and the right actions of $G$ on $S$ induce a left and a right actions (), $\bullet$ ( ) of $S$ upon itself. It follows straightforwardly that $S, S$ is a matched pair of monoids in the sense of GIM08. Let $r_{S}: S \times S \longrightarrow S \times S$ be the restriction of $r_{G}$ on $S \times S$. Then the map $r_{S}$ is bijective, non-degenerate, involutive and satisfies the braid relation. Therefore $\left(S, r_{S}\right)$ is a non-degenerate symmetric set. It follows that $\left(S, r_{S}\right)$ is a braided M3monoid in the sense of GIM08. Part (3) is straightforward. 


\section{The Retractions of a SYMmetric Group AND ITS DERIVEd CHAin OF IDEALS. SyMmETRIC GROUPS AND SYMMETRIC SETS OF FINITE MULTIPERMUTATION LEVEL}

This section is central for the paper. We study the recursive process of retraction of general symmetric groups and braces. We are particularly interested in symmetric groups, braces, and symmetric sets of finite multipermutation level. The first question to be asked is "what is the retraction of a symmetric group?" The answer is given in Subsect. 4.2 for a general symmetric group $(G, r)$. In Subsect. 4.3 we introduce the derived chain of ideals of $G$, or abbreviated $D C I$ and study its properties. Proposition 4.17 shows that DCI is an invariant of the group which gives a precise information about the recursive process of retraction on $(G, r)$. Theorem 4.21 gives new conditions equivalent to $\operatorname{mpl}(G, r)=m<\infty$. An important application of $D C I$ is Theorem 4.22 which proves that every symmetric group $(G, r)$ of finite multipermutation level $m$ is a solvable group of solvable length $\leq m$.

\subsection{Some technical results on multipermutation solution, general case.}

In this subsection we collect some notions and technical results on multipermutation solutions. We recall first an important class of solutions.

Example 4.1. Dri] (Involutive permutation solution of Lyubashenko). Let $X$ be a set of cardinality $\geq 2$, let $f \in \operatorname{Sym}(X)$. Then the map $r: X \times X \longrightarrow X \times X$ defined as

$$
r(x, y):=\left(f(y), f^{-1}(x)\right), \forall x, y \in X
$$

is a non-degenerate involutive solution of YBE, we shall refer to it as a permutation solution. Clearly, $\mathcal{L}_{x}=f$, and $\mathcal{R}_{x}=f^{-1}$, for all $x \in X$, so condition lri holds. This is a solution of multipermutation level 1. Moreover, $(X, r)$ is a (square-free) trivial solution iff $f=i d_{X}$.

The following is straightforward.

Remark 4.2. A non-degenerate symmetric set $(X, r)$ has $m p l X=1$ if and only if it is a permutation solution. The notion "a multipermutation solution" is a generalization of a permutation solution.

Let $(X, r)$, be a symmetric set of order $|X| \geq 2$. The recursive definition of retractions, $\operatorname{Ret}^{m}(X, r)=\operatorname{Ret}\left(\operatorname{Ret}^{m-1}(X, r)\right)$ implies straightforwardly that the process of taking retractions of higher order "halts" at the $m$-th step iff $m \geq 1$ is the minimal integer (if any), such that $\operatorname{Ret}^{m+1}(X, r)=\operatorname{Ret}^{m}(X, r)$. Moreover, there are obvious equivalences

$$
\begin{aligned}
{[\operatorname{mpl} X=m] } & \Longleftrightarrow\left[\operatorname{mpl}\left(\operatorname{Ret}^{(k)}(X, r)\right)=m-k, \forall k, 0 \leq k \leq m\right] \\
& \Longleftrightarrow\left[\operatorname{Ret}^{(m-1)}(X, r)\right. \text { is a permutation solution]. }
\end{aligned}
$$

In particular, if $(X, r)$ is a square-free solution, then $\operatorname{mpl} X=m$ iff $\operatorname{Ret}^{(m-1)}(X, r)$ is a trivial solution of order $\geq 2$.

Definition 4.3. Let $(X, r)$ be a symmetric set, $|X| \geq 2$. We define Condition $\left({ }^{*}\right)$ on $X$ as follows.

Condition $(*)$ : For every $x \in X$ there exist some $a \in X$, such that ${ }^{a} x=x$. 
Condition $\left(^{*}\right)$ is satisfied by every square-free solution $(X, r)$ and by every symmetric group $(G, r)$. Indeed in the first case ${ }^{x} x=x$, holds for all $x \in X$, while in the second case one has $\mathcal{L}_{1}=i d_{G}$ (condition ML0 in $G$ ).

Lemma 4.4. Let $(X, r)$ be a symmetric set, $|X| \geq 2$. Suppose that $X$ satisfies condition $\left(^{*}\right)$. Then the following hold. (1) $\mathrm{mpl} X=1 \mathrm{iff}(X, r)$ is a trivial solution. (2) One has $2 \leq \operatorname{mpl} X=m<\infty$ iff $\operatorname{Ret}^{m-1}(X, r)$ is a trivial solution with $\geq 2$ elements. (3) $\mathrm{mpl} X=2$ if and only if $(X, r)$ satisfies

$$
\mathcal{L}_{y_{x}}=\mathcal{L}_{x}, \forall x, y \in X, \quad \text { and } \mathcal{L}_{x} \neq i d_{X} \text { for some } x \in X .
$$

Moreover, in this case $(X, r)$ satisfies lri.

In particular, Lemma 4.4 is in force for symmetric groups $(G, r)$.

In cases when we have to write a sequence of successive actions in $X$ (or in $G$ ) we shall use one also well known notation

$$
\alpha \triangleright x={ }^{\alpha} x .
$$

Definition 4.5. GIC12 Let $\zeta_{1}, \zeta_{2}, \cdots, \zeta_{m} \in X$. The expression

$$
\omega=\left(\cdots\left(\left(\zeta_{m} \triangleright \zeta_{m-1}\right) \triangleright \zeta_{m-2}\right) \triangleright \cdots \triangleright \zeta_{2}\right) \triangleright \zeta_{1}
$$

is called a tower of actions (or shortly a tower) of length $m$.

Denote by $u$ the tower $\left(\cdots\left(\left(\zeta_{m} \triangleright \zeta_{m-1}\right) \triangleright \zeta_{m-2}\right) \triangleright \cdots \triangleright \zeta_{3}\right) \triangleright \zeta_{2}$, then, obviously, the element $\omega={ }^{u} \zeta_{1}$, belongs to the $G$-orbit of $\zeta_{1}$.

Remark 4.6. Suppose $(X, r)$ is a non-degenerate symmetric set, $|X| \geq 2$. Then the conditions (4.3) and (4.4) given below are equivalent:

$$
\begin{gathered}
\left(\cdots\left(\left(a \triangleright y_{m}\right) \triangleright y_{m-1}\right) \triangleright \cdots \triangleright y_{2}\right) \triangleright y_{1}=\left(\cdots\left(\left(b \triangleright y_{m}\right) \triangleright y_{m-1}\right) \triangleright \cdots \triangleright y_{2}\right) \triangleright y_{1}, \\
\forall a, b, y_{1}, \cdots y_{m} \in X .
\end{gathered}
$$

$$
\begin{gathered}
\left(\cdots\left(\left([a] \triangleright\left[y_{m}\right]\right) \triangleright\left[y_{m-1}\right]\right) \triangleright \cdots \triangleright\left[y_{3}\right]\right) \triangleright\left[y_{2}\right]=\left(\cdots\left(\left([b] \triangleright\left[y_{m}\right]\right) \triangleright\left[y_{m-1}\right]\right) \triangleright \cdots \triangleright\left[y_{3}\right]\right) \triangleright\left[y_{2}\right], \\
\forall a, b, y_{2}, \cdots y_{m} \in X .
\end{gathered}
$$

The following statement is a generalization of Theorem 5.15, GIC12 which initially was proven for square-free solutions.

Proposition 4.7. Suppose $(X, r)$ is a solution, $|X| \geq 2$.

(1) $(X, r)$ is a multipermutation solution with $\mathrm{mpl} X \leq m<\infty$, if and only if (4.3) is in force. Moreover, $\operatorname{mpl} X=m$ iff $m$ is the minimal integer for which (4.3) holds.

(2) Suppose furthermore that $X$ satisfies condition (*). Then $\operatorname{mpl} X \leq m<\infty$ iff the following is in force.

$$
\begin{gathered}
\left(\left(\cdots\left(\left(y_{m} \triangleright y_{m-1}\right) \triangleright y_{m-2}\right) \triangleright \cdots \triangleright y_{2}\right) \triangleright y_{1}\right) \triangleright x \\
\forall x, y_{1}, \cdots y_{m} \in X .
\end{gathered}
$$

Moreover, $\operatorname{mpl}(X, r)=m$ iff $m$ is the minimal integer for which 4.5) holds.

The proof is technical and we skip it here, but it can be found in GI15] Variant 2, see Proposition 5.6. 
Corollary 4.8. Let $(G, r)$ be a symmetric group. Then $\operatorname{mpl} G \leq m<\infty$ iff (4.5) is in force. Furthermore, $\operatorname{mpl}(G, r)=m$ iff $m$ is the minimal integer for which (4.5) holds.

4.2. The retraction of a symmetric group. Let $(G, r)$ be a symmetric group. Let $\Gamma=\Gamma_{l}$ be the kernel of the left action of $G$ upon itself. In a symmetric set $(X, r)$ one has $\mathcal{L}_{x}=\mathcal{L}_{y}$ iff $\mathcal{R}_{x}=\mathcal{R}_{y}$, see [ESS], section 3.2. Therefore $\Gamma$ coincides with the kernel $\Gamma_{r}$ of the right action of $G$ upon itself:

$$
\Gamma=\Gamma_{l}=\left\{a \in G \mid{ }^{a} u=u, \forall u \in G\right\}=\left\{a \in G \mid u^{a}=u, \forall u \in G\right\}=\Gamma_{r} .
$$

Moreover, $\Gamma$ is invariant with respect to the left and the right actions of $G$ upon itself. Indeed, let $a \in \Gamma, u, v \in G$. Then the equalities

$$
\left({ }^{a} u\right)\left({ }^{a} v\right)=u v={ }^{a}(u v)=\left({ }^{a} u\right)\left(a^{a} v\right)
$$

imply ${ }^{a} v={ }^{a} v=v$ for every $a \in \Gamma, u, v \in G$. Therefore $a^{u} \in \Gamma, \forall u \in G$, so $\Gamma$ is invariant with respect to the right action of $G$ upon itself. Then by Remark $3.8 \Gamma$ is $G$-invariant. It follows that $\Gamma$ is also $r$-invariant. It is clear that $\Gamma$ is an abelian normal subgroup of $G$. Let $\widetilde{G}=G / \Gamma$, be the quotient group of $G$, modulo $\Gamma$. The following result can be extracted from $[\mathrm{Ta}$, Proposition 2.9.

Fact 4.9. Ta The matched pair structure $r: G \times G \longrightarrow G \times G$ induces a map $r_{\widetilde{G}}: \widetilde{G} \times \widetilde{G} \longrightarrow \widetilde{G} \times \widetilde{G}$, which makes $\widetilde{G}$ a braided group. The pair $\left(\widetilde{G}, r_{\widetilde{G}}\right)$ is called a quotient braided group of $(G, r)$.

Note that the map $r_{\widetilde{G}}$ is in fact involutive, since $r$ is involutive. Therefore $\left(\widetilde{G}, r_{\widetilde{G}}\right)$ is a symmetric group, we call it the quotient symmetric group of $(G, r)$. We shall use notation $\widetilde{a}$ for the image of $a$ under the canonical group epimorphism $G \longrightarrow \widetilde{G}$.

Consider now $(G, r)$ as a symmetric set, with retraction $\operatorname{Ret}(G, r)=\left([G], r_{[G]}\right)$. Then the obvious implications

$$
\mathcal{L}_{a}=\mathcal{L}_{b} \Longleftrightarrow \mathcal{L}_{\left(b^{-1} a\right)}=i d_{G} \Longleftrightarrow b^{-1} a \in \Gamma \Longleftrightarrow a \Gamma=b \Gamma
$$

and an easy argument implies the following.

Lemma 4.10. Notation and assumption as above. Let $(G, r)$ be a symmetric group, let $\left(\widetilde{G}, r_{\widetilde{G}}\right)$ be its quotient symmetric group, and let $\operatorname{Ret}(G, r)=\left([G], r_{[G]}\right)$ be its retraction, where $(G, r)$ is considered as a symmetric set. Then the map

$$
\varphi:\left(\widetilde{G}, r_{\widetilde{G}}\right) \longrightarrow\left([G], r_{[G]}\right), \quad \widetilde{a} \mapsto[a],
$$

is an isomorphism of symmetric sets. Moreover, the retraction $\left([G], r_{[G]}\right)$ is also a symmetric group and $\varphi$ is an isomorphism of symmetric groups.

We shall often identify the retraction $\operatorname{Ret}(G, r)=\left([G], r_{[G]}\right)$ of $(G, r)$ and its quotient symmetric group $\left(\widetilde{G}, r_{\widetilde{G}}\right)$, where $\widetilde{G}=G / \Gamma$.

Remark 4.11. Analogous results but in terms of braces, (and the socle of a brace) were proven in works of Rump some years after Takeuchi, see [Ru07, Proposition 7 , for details. One can find helpful interpretation of some Rump's results and other results on braces in CJO14. 
4.3. The derived chain of ideals of a symmetric group. Let $(G,+, \cdot)$ be a left brace. Recall that a subset $I \subset G$ is called an ideal of $G$ if it is (i) a normal subgroup of the multiplicative group $(G, \cdot)$, (ii) a subgroup of the additive group of $(G,+)$, and (iii) $I$ is invariant with respect to the left actions $\mathcal{L}_{a}, a \in G$, CJO14.

Suppose now that $(G, r)$ is a symmetric group, $(G,+, \cdot)$ is the associated left brace, and $H$ is a subgroup of $(G, r)$. By Remark $3.8 H$ is left $G$-invariant iff $H$ is $G$ invariant, hence $H$ is also $r$-invariant. Moreover, in this case $H$ is (automatically) a subgroup of the additive group $(G,+)$ of the brace. Indeed, the equalities $a-b=$ $a+{ }^{b}\left(b^{-1}\right)=a\left({\left(a^{-1} b\right)}\left(b^{-1}\right)\right)$ imply that $a-b \in H$, whenever $a, b \in H$. So a subgroup $H$ of $(G, r)$ is an ideal of the brace $(G,+, \cdot)$ if and only if it is a $G$-invariant normal subgroup of $(G, r)$.

Definition 4.12. We say that $H$ is an ideal of the symmetric group $(G, r)$ if $H$ is a normal subgroup of $G$ which is $G$-invariant.

Suppose $H$ is an ideal of the symmetric group $(G, r)$. Then the (multiplicative) quotient $\bar{G}=G / H$ has also a canonical symmetric group structure $\left(\bar{G}, r_{\bar{G}}\right)$ induced from $(G, r)$. Analogously, (and independently) there is a canonical brace structure $\left(\overline{G_{b r}},+, \cdot\right)$ on the quotient brace $\overline{G_{b r}}=(G,+, \cdot) / H$ modulo the (brace) ideal $H$. Note that the left brace $(\bar{G},+, \cdot)$ associated with the quotient symmetric group $\left(\bar{G}, r_{\bar{G}}\right)$ (by Definition-Convention 3.7 ) and the quotient brace $\overline{G_{b r}}$ coincide, so we shall simply use the notation $(\bar{G},+, \cdot)$.

The following Isomorphism Theorems for Symmetric Groups are in force. The proofs are routine and we leave them for the reader.

Remark 4.13. (1) [First Isomorphism Theorem for Symmetric Groups] Let $f:(G, r) \longrightarrow\left(\widetilde{G}, r_{\widetilde{G}}\right)$ be an epimorphism of symmetric groups. The kernel $K=\operatorname{ker} f$ is an ideal of $(G, r)$, and there is a natural isomorphism of symmetric groups $G / K \simeq \widetilde{G}$.

(2) [Third Isomorphism Theorem for Symmetric groups] Let $(G, r)$ be a symmetric group, let $K$ be an ideal of $G, \widetilde{G}=G / K$, and let $f: G \longrightarrow \widetilde{G}$ be the canonical epimorphism of symmetric groups (one has $\operatorname{ker} f=K$ ).

(a) There is a bijective correspondence

\{ideals $H$ of $G$ containing $K\} \longleftrightarrow$ ideals $\widetilde{H}$ of $\widetilde{G}$ \}, given by

$$
H \longmapsto f(H) \simeq H / K, \quad f^{-1}(H) \longleftarrow \widetilde{H} .
$$

(b) For every ideal $H \supset K$ of $G$ one has

$$
(G / K) /(H / K) \simeq G / H, \quad g K .(H / K) \mapsto g H .
$$

Analogous statements are in force for braces.

Suppose $(G, r)$ is a symmetric group, then the kernel $\Gamma$ of the left action of $G$ upon itself, see (4.6), is a $G$-invariant normal subgroup of $G$, so it is an ideal, called the socle of $G$, and denoted $\operatorname{Soc}(G)$. Clearly, $\operatorname{Soc}(G)$ is an abelian subgroup of $G$, $(\operatorname{Soc}(G)=\{1\}$ is also possible). Moreover, $\operatorname{Soc}(G)=G$ iff $(G, r)$ is the trivial solution and $G \neq \operatorname{Soc}(G)=\{1\}$ iff $(G, r)$ is not retractable. 
We call $(G, r)$ a prime symmetric group if $G$ does not have proper, nontrivial ideals. Prime braces are defined analogously.

Remark 4.14. Suppose $(G, r)$ is a prime symmetric group. Then either (i) $\operatorname{Soc} G=$ $G$, thus $(G, r)$ is the trivial solution, or (ii) Soc $G=\{1\}$, hence $G$ is not retractable. More generally, if $K$ is a maximal ideal of a symmetric group $(G, r)$, then the quotient symmetric group $\widetilde{G}=G / K$ is either a trivial solution, or $\widetilde{G}$ can not be retracted.

Remark 4.15. Let $G=(G, r)$ be a symmetric group, denote by $\left(G^{j}, r^{j}\right), j \geq 0$, its $j$-th retraction. So for each $j \geq 1$ one has $\left(G^{j}, r^{j}\right)=\operatorname{Ret}^{j}(G, r) \simeq G^{j-1} / \Gamma_{j}$, where $\Gamma_{j}=\operatorname{Soc}\left(G^{j-1}\right)$. We have $G^{0}=\operatorname{Ret}^{0}(G, r)=G, \Gamma_{1}=\Gamma$. For $j \geq 0$ denote by $\varphi_{j+1}$ the canonical epimorphism of symmetric groups

$$
G^{j} \stackrel{\varphi_{j+1}}{\longrightarrow} G^{j} / \Gamma_{j+1} \simeq G^{j+1}, \quad \operatorname{ker} \varphi_{j+1}=\Gamma_{j+1} .
$$

Observe the canonical sequence of epimorphisms of symmetric groups (some of these possibly coincide):

$$
G \stackrel{\varphi_{1}}{\longrightarrow} G^{1}=G / \Gamma \stackrel{\varphi_{2}}{\longrightarrow} G^{2}=G^{1} / \Gamma_{2} \stackrel{\varphi_{3}}{\longrightarrow} G^{3}=G^{2} / \Gamma_{3} \stackrel{\varphi_{4}}{\longrightarrow} \cdots .
$$

Set $K_{0}=\{1\}, K_{1}=\Gamma=\Gamma_{1}$, and for all $j>1$ denote by $K_{j}$ the pull-back of $\Gamma_{j}$ in $G$, that is $K_{j}=\left(\varphi_{j-1} \circ \cdots \varphi_{2} \circ \varphi_{1}\right)^{-1}\left(\Gamma_{j}\right)$. Each set $K_{j}, j \geq 0$, is an ideal of $G$, thus we obtain a (non-decreasing) chain of ideals in $G$ :

$$
\{1\}=K_{0} \subset K_{1} \subset K_{2} \subset \cdots \subset K_{j} \subset \cdots .
$$

Definition 4.16. We shall call the chain (4.7) the derived chain of ideals of the symmetric group $(G, r)$ or shortly, the derived chain of $G$.

The derived chain is an invariant of the symmetric group $G$ which reflects the recursive process of retraction. In particular, it gives an explicit information whether $(G, r)$ has a finite multipermutation level.

Proposition 4.17. Let $(G, r)$ be a symmetric group with derived chain of ideals (4.7). Notation as above.

(1) For all $j \geq 1$ there are isomorphisms

$$
K_{j} / K_{j-1} \simeq \Gamma_{j}, \quad G / K_{j} \simeq \operatorname{Ret}^{j}(G, r),
$$

and canonical epimorphisms of symmetric groups

$$
\mu_{j}: G / K_{j-1} \longrightarrow G / K_{j}, \text { ker } \mu_{j} \simeq K_{j} / K_{j-1} .
$$

In particular, $K_{j} / K_{j-1}=\operatorname{Soc}\left(G / K_{j-1}\right), j \geq 1$, are abelian symmetric groups $\left(K_{j} / K_{j-1}=1\right.$ is possible). The following diagram is commutative:

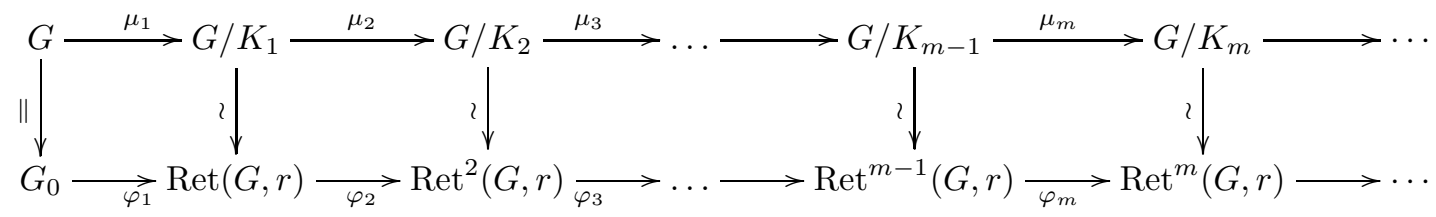

(The vertical arrows denote isomorphisms of symmetric groups).

(2) The derived chain of $G$ stabilizes if and only if $\operatorname{Ret}^{j+1}(G, r)=\operatorname{Ret}^{j}(G, r)$ (or equivalently, $K_{j+1}=K_{j}$ ) for some $j \geq 0$. 
(3) Let $m$ be the minimal integer (if any) such that $K_{m+1}=K_{m}$. Then the derived chain has length $m+1$, $\operatorname{Ret}^{m}(G, r)=\operatorname{Ret}^{m+1}(G, r)$, so the process of recursive retraction halts in $m$ steps. Moreover, exactly one of the following two conditions is satisfied:

(a) $K_{m}=G$. Then $m \geq 1$, and $(G, r)$ is a multipermutation solution with $\operatorname{mpl}(G, r)=m$

(b) $K_{m} \varsubsetneqq G$ ( $m=0$ is possible). Then $\operatorname{Ret}^{m}(G, r)$ is a symmetric group of order $\geq 2$ which can not be retracted.

Proof. (1) We "iterate" the Third Isomorphism Theorem for symmetric groups to yield the following:

$$
\begin{array}{ll}
\Gamma_{1}=K_{1}, & \operatorname{Ret}^{1}(G, r)=\left(G^{1}, r^{1}\right) \simeq G / K_{1}, \\
\Gamma_{2} \simeq K_{2} / K_{1}, & \operatorname{Ret}^{2}(G, r)=\left(G^{2}, r^{2}\right) \simeq G / K_{2}, \\
\Gamma_{j} \simeq K_{j} / K_{j-1}, & \operatorname{Ret}^{j}(G, r)=\left(G^{j}, r^{j}\right) \simeq G / K_{j}, \\
& \forall j \geq 1 .
\end{array}
$$

The isomorphisms $\Gamma_{j} \simeq K_{j} / K_{j-1}$ imply that each of the quotient groups $K_{j} / K_{j-1}$, $j \geq 1$, is abelian. The commutativity of the diagram is straightforward.

(2) The above argument implies that there is an equality $K_{j+1}=K_{j}$ iff $\Gamma_{j+1}=1$, which is equivalent to $\operatorname{Ret}^{j+1}(G, r)=\operatorname{Ret}^{j}(G, r)$. In this case one has $\operatorname{Ret}^{j+p}(G, r)=$ $\operatorname{Ret}^{j}(G, r)$, therefore $K_{j+p}=K_{j}$, for every $p \geq 1$, and the derived chain stabilizes. Suppose that this is the case and let $m$ be the minimal integer with $K_{m+1}=K_{m}$. Without loss of generality we may assume $m \geq 1$. Then the derived chain of $G$ is exactly $\{1\}=K_{0} \varsubsetneqq K_{1} \varsubsetneqq \cdots \varsubsetneqq K_{m}$. It follows from our discussion above that all retractions $\operatorname{Ret}^{j}(G, r), 0 \leq j \leq m$ are distinct, but $\operatorname{Ret}^{m}(G, r)=\operatorname{Ret}^{m+p}(G, r), \forall p \geq 1$, therefore the process of recursive "retraction" halts exactly at the $m$-th step.

(3) (a). Assume $K_{m}=G$ is in force. Then $m>1$, the symmetric group $G^{m-1}=$ $\operatorname{Ret}^{m-1}(G, r) \simeq G / K_{m-1}$ has order $>1$ and $\Gamma_{m}=\operatorname{Soc}\left(G^{m-1}\right) \simeq K_{m} / K_{m-1}$, $G / K_{m-1}=G^{m-1}$. Clearly, then $\operatorname{Ret}\left(G^{m-1}\right)=\operatorname{Ret}^{m}(G, r)$ is a one element solution, so $\operatorname{mpl}(G, r)=m$.

(b). Suppose now $K^{m} \varsubsetneqq G$ holds. In this case $\operatorname{Ret}^{m}(G, r) \simeq G / K_{m}$ is a symmetric

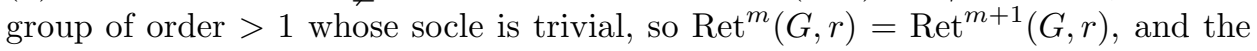
process of "retracting" $(G, r)$ halts in $m$ steps, but without reaching a one element solution. Clearly, in this case $(G, r)$ is not a multipermutation solution.

Corollary 4.18. The derived chain of ideals of $G$ has the shape

$$
\{1\}=K_{0} \varsubsetneqq K_{1} \varsubsetneqq K_{2} \varsubsetneqq \cdots \varsubsetneqq K_{m-1} \varsubsetneqq K_{m}=G
$$

if and only if $K_{m-1} \varsubsetneqq K_{m}=G$.

Let $B=(B,+, \cdot)$ be a left brace, the operation $*$ on $B$ is defined via (3.4). By $B^{(s)}, s=1,2, \cdots$, we denote the chain of ideals introduced by Rump in Ru07, one has $B^{(1)}=B$ and $B^{(s+1)}=B^{(s)} * B$. $B$ is called a right nilpotent brace if there exists a positive integer $n$ such that $B^{(n)}=0$, CGIS16. Using (3.5) and (3.4) one can present the usual left action of $B$ upon itself as

$$
{ }^{a} b=a * b+b, \quad a * b={ }^{a} b-b .
$$


So the socle of $B$ satisfies

$$
\operatorname{Soc}(B) * B=0, \quad \operatorname{Soc}(B)=\{a \in B \mid a * b=0, \forall b \in B\} .
$$

Remark 4.19. Suppose $(B,+, \cdot)$ is a left brace, $|B|>1$. Then $B * B=0$ if and only if $\operatorname{mpl} B=1$. In this case $a \cdot b=a+b, \forall a, b \in B$ and $B$ is a two-sided brace.

Remark 4.20. The following theorem provides four different conditions, each of which is equivalent to $\operatorname{mpl}(G, r)=m$. The equivalence of conditions (2a) and (2d) was proved first by the author for the case when $(G,+, \cdot)$ is a two-sided brace, see Proposition 5.16, GI15. Later this equivalence was proven for the general case when $(G,+, \cdot)$ is an arbitrary left brace, CGIS16], Proposition 6. Using our technique with derived chains we provide an independent (and different) proof of this particular equivalence and find new equivalent conditions.

Theorem 4.21. Let $(G, r)$ be a nontrivial symmetric group,$(G,+, \cdot)$ its associated left brace, and let $\{1\}=K_{0} \subseteq K_{1} \subseteq K_{2} \subseteq \cdots$ be its derived chain of ideals.

(1) The derived chain of ideals satisfy

$$
\left(\left(\cdots \left((K_{j} * \overbrace{G) * G) * \cdots) * G)}^{s \text { times }} \subseteq K_{j-s}, \forall j, s, 1 \leq s \leq j .\right.\right.\right.
$$

(2) The following conditions are equivalent.

(a) $(G, r)$ has a finite multipermutation level $\operatorname{mpl} G=m \geq 1$.

(b) The derived chain of ideals of $G$ has the shape [4.9).

(c) The ideals $G^{(j)}$ satisfy:

$$
G^{(j+1)} \subseteq K_{m-j}, 0 \leq j \leq m ; \quad G^{(j+1)} \varsubsetneqq K_{m-j-1}, 0 \leq j \leq m-1 .
$$

(d) The brace $G$ is right nilpotent of nilpotency class $m+1$, i.e. $G^{(m+1)}=$ 0 , and $G^{(m)} \neq 0$.

Proof. We shall use the notation and results of Proposition 4.17 Recall first that $1=0$ in $(G,+, \cdot)$, so we also have $\{0\}=K_{0}$.

(1). We have shown that for each $j \geq 0$ there are isomorphisms of symmetric groups

$$
\operatorname{Ret}^{j}(G, r)=G^{j} \simeq G / K_{j}, \operatorname{Soc}\left(G / K_{j}\right)=K_{j+1} / K_{j},
$$

where for completeness $\operatorname{Ret}^{0}(G, r)=G^{0}=G$. In each of the braces $\left(G^{j},+, \cdot\right)$ we also have the induced operation $*$. Then the first equality in (4.11) implies $\left(\operatorname{Soc}\left(G / K_{j}\right)\right) *\left(G / K_{j}\right)=0$, or equivalently, $\left(K_{j+1} / K_{j}\right) *\left(G / K_{j}\right)=0$ holds in $G / K_{j}$. This implies $K_{j+1} * G \subseteq K_{j}$, for each $j \geq 0$, which verifies (4.12), where $s=1$. The general equalities (4.12), where $s$ is an integer, $1 \leq s \leq j$, follows by induction on $s$.

(2). (2a) $\Longrightarrow(2 \mathrm{~b})$. Assume $\operatorname{mpl} G=m$. Then (4.14) implies that (4.9) is exactly the derived chain of ideals for $G$.

(2b) $\Longrightarrow$ (2a). Suppose the derived chain of $G$ is given by (4.9), then $\operatorname{Ret}^{m-1}(G, r) \simeq$ $G / K_{m-1}$ is a solution of order $\geq 2$, and $\operatorname{Ret}^{m}(G, r) \simeq G / K_{m}=\{1\}$ is a one element solution, therefore $\operatorname{mpl} G=m$. 
(2c) $\Longrightarrow$ (2b). Setting $j=0$ in (4.13) we get $G=G^{(1)}=K_{m} \varsubsetneqq K_{m-1}$, but by definition $K_{m} \supseteq K_{m-1}$, so $K_{m-1} \varsubsetneqq K_{m}$ and by Corollary 4.18 the derived chain of ideals has the shape (4.9).

$(2 \mathrm{~b}) \Longrightarrow(2 \mathrm{C})$. Assume now the derived chain has the shape (4.9). Then $G^{(1)}=K_{m}$, and the equalities (4.12) imply

$$
G^{(j+1)}=\left(\left(\cdots \left((K_{m} * \overbrace{G) * G) * \cdots) * G)}^{j \text { times }} \subseteq K_{m-j}, \forall j, 1 \leq j \leq m .\right.\right.\right.
$$

We shall use induction on $m$ to prove $G^{(j+1)} \varsubsetneqq K_{m-j-1}, \forall j, 1 \leq j \leq m$. If $m=1$, the derived chain is trivial: $\{1\}=K_{0} \varsubsetneqq K_{1}=G$, so clearly $G^{(1)}=K_{1}$, and $G^{(1)} \subsetneq K_{0}$, which gives the base for induction. Assume for all $m, 1 \leq m \leq n$, (4.9) implies

$$
G^{(j+1)} \varsubsetneqq K_{m-j-1}, \forall j 0 \leq j \leq m .
$$

Suppose the derived chain of ideals has length $m+1$ and shape (4.9) with $m=n+1$. We consider the quotient group $\widetilde{G}=G / K_{1}$. Then the ideals of the derived chain of $\widetilde{G}$ are exactly $\widetilde{K_{s}}=K_{s} / K_{1}, 1 \leq s \leq m$, so the chain has length $m=n+1$ and shape

$$
\{1\}=\widetilde{K_{1}}=K_{1} / K_{1} \varsubsetneqq \widetilde{K_{2}}=K_{2} / K_{1} \varsubsetneqq \cdots \varsubsetneqq \widetilde{K_{m-1}} \varsubsetneqq \widetilde{K_{m}}=\widetilde{G}
$$

By the inductive assumption $\widetilde{G}^{(j+1)} \mp \widetilde{K_{n-j-1}}$ for all $j, 1 \leq j \leq n$. This implies $G^{(j+1)} \varsubsetneqq K_{n-j-1}$, hence (4.15) is in force.

Next we shall show $(2 \mathrm{C}) \Longrightarrow(2 \mathrm{~d}) \Longrightarrow(2 \mathrm{~b})$ using again "the derived cains technique". The implication (2c) $\Longrightarrow(2 \mathrm{~d}$ ) is straightforward.

$(2 \mathrm{~d}) \Longrightarrow(2 \mathrm{~b})$. Assume $G^{(m+1)}=0$, and $G^{(m)} \neq 0$. We shall use induction on $m$ to show $K_{m-1} \subsetneq K_{m}=G$. The following implications are clear:

$$
G^{(m+1)}=0 \Longleftrightarrow G^{(m)} \subseteq K_{1}, \quad G^{(m)} \neq 0 \Longleftrightarrow G^{(m-1)} \varsubsetneqq K_{1} .
$$

If $m=1$ then the equalities $G * G=G^{(2)}=0$, and $G=G^{(1)} \neq 0$ imply $G=$ Soc $G=K_{1} \supseteq K_{0}$ which gives the base for induction. Assume our statement is true for all $m, 1 \leq m \leq n-1$. Suppose $G^{(m+1)}=0$, and $G^{(m)} \neq 0$, where $m=n$. Consider the quotient group $\widetilde{G}=G / K_{1}$ and its derived chain of ideals $\{1\}=\widetilde{K_{1}}=K_{1} / K_{1} \subseteq \widetilde{K_{2}}=K_{2} / K_{1} \subseteq \cdots$. Our assumption and (4.16) imply $\widetilde{G}^{(m)}=0$, and $\widetilde{G}^{(m-1)} \neq 0$. Thus by the inductive hypothesis the derived chain of ideals of $\widetilde{G}$ has length $m$ and $\widetilde{K_{m-1}}=K_{m-1} / K_{1} \subsetneq \widetilde{K_{m}}=K_{m} / K_{1}=\widetilde{G}$. By the Third Isomorphism Theorem for symmetric groups this implies $K_{m-1} \subsetneq K_{m}=G$, hence by Corollary 4.18 the derived chain of $G$ satisfies (4.9).

Theorem 4.22. Every symmetric group $(G, r)$ of finite multipermutation level $\operatorname{mpl} G=m$ is a solvable group of solvable length $\operatorname{sl} G \leq m$.

Proof. By Theorem 4.21 $\mathrm{mpl} G=m$ implies that $G$ has a chain of normal subgroups given by (4.9). Moreover, by Proposition 4.17 each quotient $K_{j} / K_{j-1}, 1 \leq j \leq m$, is abelian. It follows then that $G$ is a solvable group with solvable length sl $G \leq m$. 
Remark 4.23. Note that solvability of a symmetric group $(G, r)$ does not imply $\operatorname{mpl}(G, r)<\infty$. Recall that the symmetric group $G=G(X, r)$ is solvable, whenever $(X, r)$ is a finite solution, [ESS. Suppose that the solution $(X, r)$ is finite but $(X, r)$ is not a mulipermutation solution, e.g. Vendramin's example of order 8 , see Remark 5.12. Then the associated group $G=G(X, r)$ is a solvable group which is not a multipermutation solution (this follows from Theorem 5.15).

5. The symmetric group $G(X, r)$ OF a Symmetric Set $(X, r)$. Derived SYMMETRIC GROUPS AND DERIVED PERMUTATION GROUPS OF A SOLUTION

In this section as usual $(X, r)$ is a non-degenerate symmetric set of arbitrary cardinality, $G=G(X, r)=\left(G, r_{G}\right),(G,+, \cdot), \mathcal{G}=\mathcal{G}(X, r), \Gamma=\operatorname{Soc} G, K=\operatorname{ker} \mathcal{L}$, see Definition 2.5.

\subsection{The symmetric group $G(X, r)$ of a symmetric set $(X, r)$ and its re- traction.}

Remark 5.1. [Ta, p. 15. Let $(X, r)$ be a (non-degenerate) symmetric set, notation as above. Then the following conditions hold. (i) The kernels $\Gamma$ and $K$ coincide, so:

$$
\begin{aligned}
\Gamma & =\Gamma_{l}=\left\{a \in G \mid{ }^{a} u=u, \forall u \in G\right\}=\Gamma_{r}=\left\{a \in G \mid u^{a}=u, \forall u \in G\right\} \\
& =K=\left\{a \in G \mid{ }^{a} x=x, \forall x \in X\right\} .
\end{aligned}
$$

(ii) $\Gamma=K$ is an abelian normal subgroup of $G$. (iii) $\widetilde{G}=G / \Gamma$ is a quotient symmetric group of $\left(G, r_{G}\right)$, in the sense of Takeuchi, so $\operatorname{Ret}\left(G, r_{G}\right) \simeq \widetilde{G}=G / \Gamma$ is an isomorphism of symmetric groups.

Lemma 4.10 and the equality of the two kernels $\Gamma=K$ imply the following.

Corollary 5.2. Let $(X, r)$ be a non-degenerate symmetric set, notation as above.

(1) There is an equality $\operatorname{Soc} G=K$ and an isomorphism of symmetric groups

$$
\operatorname{Ret}\left(G, r_{G}\right) \simeq \mathcal{G}(X, r) .
$$

(2) If $\operatorname{mpl}\left(G, r_{G}\right)=m<\infty$ then

$$
m=\operatorname{mpl}\left(G, r_{G}\right) \geq \operatorname{mpl} G(\operatorname{Ret}(X, r)) \geq \operatorname{mpl}\left(\mathcal{G}, r_{\mathcal{G}}\right)=m-1 .
$$

(3) If the solution $(X, r)$ is finite then the retraction $\operatorname{Ret}\left(G, r_{G}\right) \simeq \mathcal{G}(X, r)$ is a finite symmetric group.

The equality $\Gamma=K$ for the symmetric groups $G=G(X, r)$ was published first by Takeuchi in 2003, ([Ta], see p. 15). A natural questions arises.

How can we express the higher retractions $\operatorname{Ret}^{j}\left(G, r_{G}\right), j \geq 2$ ? In particular, what is the retraction $\operatorname{Ret}\left(\mathcal{G}, r_{\mathcal{G}}\right) \simeq \operatorname{Ret}^{2}\left(G, r_{G}\right)$ ?

We shall answer this questions in terms of derived permutation groups of $(X, r)$, see Definition 5.9. Lemma 5.6 and Theorem 5.8. 
5.2. Derived symmetric groups and derived permutation groups of a solution. In assumption and conventions as above we collect a list of notation which will be used throughout the paper. The existence of all objects and maps given below is proven in Proposition 5.7. Here $j \geq 0$ is an integer.

\section{Notation 5.3.}

We set $\operatorname{Ret}^{0}(X, r)=(X, r), \operatorname{Ret}^{j}(X, r)=\operatorname{Ret}\left(\operatorname{Ret}^{j-1}(X, r)\right)$ is the $j$-th retraction of $(X, r), j \geq 1$, but when $j=1$ we use both notations $\operatorname{Ret}(X, r)=\operatorname{Ret}^{1}(X, r)$ and $\left([X], r_{[X]}\right)$.

$x^{(j)}$ denotes the image of $x$ in $\operatorname{Ret}^{j}(X, r)\left(x^{(0)}=x\right)$.

$G_{j}:=G\left(\operatorname{Ret}^{j}(X, r)\right), G_{0}=G(X, r)=G ; \mathcal{G}_{j}:=\mathcal{G}\left(\operatorname{Ret}^{j}(X, r)\right), \mathcal{G}_{0}=\mathcal{G}(X, r)=\mathcal{G}$.

$\mathcal{L}^{j}: G_{j} \longrightarrow \mathcal{G}_{j}$ is the epimorphism extending the assignment $x^{(j)} \mapsto \mathcal{L}_{x^{(j)}} \in$ $\operatorname{Sym}\left(\operatorname{Ret}^{j}(X, r)\right), x \in X, \mathcal{L}^{0}=\mathcal{L}: G \longrightarrow \mathcal{G}$, extends $x \mapsto \mathcal{L}_{x}, x \in X$.

$\mathcal{K}_{j}$ is the pull-back of $\operatorname{ker} \mathcal{L}^{j}$ in $G$, in particular $\mathcal{K}_{0}=\operatorname{ker} \mathcal{L}$.

$\nu_{j}: G_{j} \longrightarrow G_{j+1}$ is the epimorphism extending the assignment $x^{(j)} \mapsto x^{(j+1)} . N_{j}$ is the pull-back of $\operatorname{ker} \nu_{j}$ in $G, N_{0}=\operatorname{ker} \nu_{0}$.

$\varphi_{j}: \mathcal{G}_{j} \longrightarrow \mathcal{G}_{j+1}$ is the epimorphism extending the assignments $\mathcal{L}_{x^{(j)}} \mapsto \mathcal{L}_{x^{(j+1)}}, x \in$ $X$, see Proposition 5.7. $H_{j}$ is the pull-back of $\operatorname{ker} \varphi_{j}$ in $G$.

Lemma 5.4. In assumption and notation as above the following conditions hold.

(1) The canonical epimorphism of solutions $\nu_{0}:(X, r) \longrightarrow\left([X], r_{[X]}\right), x \mapsto[x]$, extends to a group epimorphism $\nu_{0}: G_{0} \longrightarrow G_{1}$. Analogously, for each $j \geq 1$ there exists an epimorphism of symmetric groups

$$
\nu_{j}:\left(G_{j}, r_{G_{j}}\right) \longrightarrow\left(G_{j+1}, r_{G_{j+1}}\right) .
$$

(2) There is a canonical epimorphism of symmetric groups

$$
\varphi_{0}: \mathcal{G}_{0} \longrightarrow \mathcal{G}_{1}, \quad \mathcal{L}_{x} \mapsto \mathcal{L}_{[x]}, \forall x \in X
$$

(3) The subgroups $N_{0}, \mathcal{K}_{0}=K, \mathcal{K}_{1}, H_{0}$ of $G$ are ideals of the symmetric group $\left(G, r_{G}\right)$. One has

$$
\begin{aligned}
N_{0} \varsubsetneqq \mathcal{K}_{0} \subset \mathcal{K}_{1}=H_{0} \\
\operatorname{ker} \nu_{1} \simeq N_{1} / N_{0} ; \quad \operatorname{ker} \mathcal{L}^{1} \simeq \mathcal{K}_{1} / N_{0} ; \quad \operatorname{ker} \varphi_{0} \simeq \mathcal{K}_{1} / \mathcal{K}_{0} .
\end{aligned}
$$

In particular, $N_{0}$ and $\mathcal{K}_{0}$ are abelian.

(4) There is a canonical epimorphism of symmetric groups

$f_{0}: G_{1} \longrightarrow \mathcal{G}_{0},[x] \mapsto \mathcal{L}_{x}, x \in X$, where ker $f_{0} \simeq \mathcal{K}_{0} / N_{0}$, and $f_{0} \circ \nu_{0}=\mathcal{L}^{0}$.

(5) There are short exact sequences:

$$
\begin{array}{rl}
1 \longrightarrow N_{0} \longrightarrow G \stackrel{\nu_{0}}{\longrightarrow} G_{1} \longrightarrow 1 & 1 \longrightarrow \mathcal{K}_{0} \longrightarrow G \stackrel{\mathcal{L}^{0}}{\longrightarrow} \mathcal{G}_{0} \longrightarrow 1 \\
1 \longrightarrow N_{1} / N_{0} \longrightarrow G_{1} \stackrel{\nu_{1}}{\longrightarrow} G_{2} \longrightarrow 1 & 1 \longrightarrow \mathcal{K}_{0} / N_{0} \longrightarrow G_{1} \stackrel{f_{0}}{\longrightarrow} \mathcal{G}_{0} \longrightarrow 1 \\
1 \longrightarrow K_{1} / N_{0} \longrightarrow G_{1} \stackrel{\mathcal{L}^{1}}{\longrightarrow} \mathcal{G}_{1} \longrightarrow 1 & 1 \longrightarrow \mathcal{K}_{1} / \mathcal{K}_{0} \longrightarrow \mathcal{G} \stackrel{\varphi_{0}}{\longrightarrow} \mathcal{G}_{1} \longrightarrow 1
\end{array}
$$

(6) The following diagram is commutative: 


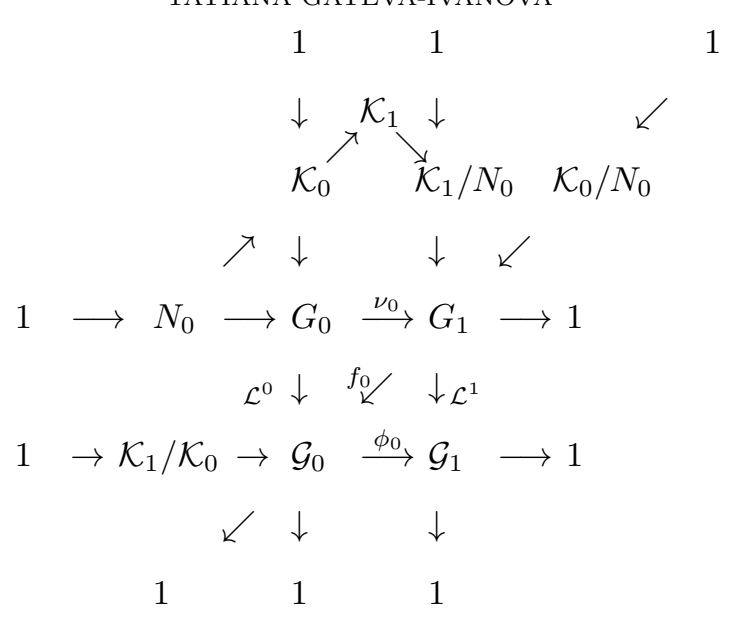

Proof. (11). The canonical map $\nu_{0}: X \longrightarrow[X], x \mapsto[x]$, from $X$ to its retract is $a$ braiding-preserving map, (epimorphism of solutions) therefore, by [LYZ, Proposition 6 , its canonical extension $\nu_{0}: G \longrightarrow G_{1}$ is an epimorphism of symmetric groups (that is a braiding preserving group epimorphism) $\nu_{0}:\left(G, r_{G}\right) \longrightarrow\left(G_{1}, r_{G_{1}}\right)$. Condition (2) is clear. We shall verify (3). We know that $K=\mathcal{K}_{0}=\Gamma=\operatorname{Soc} G$, so it is an ideal of $G$. The kernel $N=N_{0}=\operatorname{ker} \nu_{0}$ is a normal subgroup of $G$ which consists of all $a \in G$, such that $[a]=1_{G_{1}}$. Suppose $a \in N$. One has $\left[{ }^{u} a\right]={ }^{[u]}[a]={ }^{[u]} 1_{G_{1}}=1_{G_{1}}$ hence $N$ is left $G$-invariant, and therefore $N$ is an ideal of $G$. To verify $N_{0} \subset K$ it will be enough to show the implication

$$
[a]=1_{G_{1}} \Longrightarrow \mathcal{L}_{a}=\mathrm{id}_{X}
$$

Note first that the retraction $[X]$ is embedded in $G_{1}$, and $[x] \neq 1_{G_{1}}$, for all $x \in X$. Suppose $a \in N, a \neq 1$. Then $a$ has a reduced length $|a| \geq 2$, and can be written as $a=u v$, where $u, v \in G,|u|,|v| \geq 1$. One has $[a]=[u v]=[u][v]=1_{G_{1}}$, hence $[u]=[v]^{-1}=\left[v^{-1}\right]$. This gives $[a]=\left[v^{-1}\right][v]$. Let $x \in X$, one has ${ }^{a} x=v^{-1}\left({ }^{v} x\right)=x$. Therefore ${ }^{a} x=x$, for every $x \in X$, so $\mathcal{L}_{a}=\operatorname{id}_{X}$. This verifies $N_{0} \subseteq \mathcal{K}_{0}$. The socle $\Gamma=\mathcal{K}_{0}$ is an abelian group and so is $N_{0}$.

The kernel $\widetilde{\mathcal{K}_{1}}$ of the map $\mathcal{L}^{1}: G_{1} \longrightarrow \mathcal{G}_{1}$ is the socle of $G_{1}$, hence, an ideal of $G_{1}$. But, by definition $\mathcal{K}_{1}=\nu_{0}^{-1}\left(\widetilde{\mathcal{K}_{1}}\right)\left(\widetilde{\mathcal{K}_{1}}=\nu_{0}\left(\mathcal{K}_{1}\right)\right)$ so by the Third Isomorphism theorem, $\mathcal{K}_{1}$ is also an ideal of $(G, r)$, which contains $N_{0}$. The equality $H_{0}=\mathcal{K}_{1}$ follows from the implications:

$$
\begin{aligned}
u \in H_{0} & \Longleftrightarrow \mathcal{L}_{[u]}=\operatorname{id}_{[X]} \quad \Longleftrightarrow{ }^{[u]}[x]=\left[{ }^{u} x\right]=[x], \quad \forall x \in X \\
& \Longleftrightarrow{ }^{\left({ }^{u} x\right)} z={ }^{x} z, \quad \forall x, z \in X \Longleftrightarrow \mathcal{L}_{\left({ }^{u} x\right)}=\mathcal{L}_{x}, \quad \forall x \in X \\
& \Longleftrightarrow u \in \mathcal{K}_{1} .
\end{aligned}
$$

Now the inclusions (5.3) for the three kernels are clear. This implies the second line in (5.3). The existence of the short exact sequences (5.4) is straightforward from (5.3). It is easy to see that (3), (4), and (6) are in force.

Remark 5.5. Suppose $(X, r)$ is a solution of finite order, which is not a permutation solution ( $\mathrm{mpl} X \geq 2$ is not necessarily finite). Then $K_{0}$ is a normal subgroup of $G$ of finite index $p=\left[G: K_{0}\right]$. In contrast, the index $\left[G: N_{0}\right]$ of $N_{0}$ is not finite. Indeed, the retraction $\left([X], r_{[X]}\right)$ is a solution of order $>1$, which generates the 
group $G_{1}=G\left([X], r_{[X]}\right)$. Note that $[x] \neq 1_{G_{[X]}}$, for all $x \in X$. The group $G_{1}$ is torsion free as a YB group of a solution of order $>1$. In particular, $\left[x^{p}\right]=[x]^{p} \neq 1_{G_{[X]}}$, so $x^{p}$ is not in $N_{0}, \forall x \in X$. On the other hand $x^{p} \in \mathcal{K}_{0}, \forall x \in X$.

Lemma 5.6. There is an isomorphism of symmetric groups:

$$
\operatorname{Ret}\left(\mathcal{G}, r_{\mathcal{G}}\right) \simeq \mathcal{G}(\operatorname{Ret}(X, r)), \quad \text { where } \operatorname{Soc}(\mathcal{G}) \simeq \mathcal{K}_{1} / K_{0}
$$

Proof. Recall that $\mathcal{G}(\operatorname{Ret}(X, r))$ was denoted as $\left(\mathcal{G}_{1}, r_{\mathcal{G}_{1}}\right)$. We shall prove that $\operatorname{Soc}(\mathcal{G})=\operatorname{ker} \varphi_{0}$, where $\varphi_{0}: \mathcal{G}_{0} \longrightarrow \mathcal{G}_{1}, \quad \mathcal{L}_{x} \mapsto \mathcal{L}_{[x]}$, is the canonical epimorphism from Lemma [5.4. This follows from the implications

$$
\begin{aligned}
& \mathcal{L}_{u} \in \operatorname{Soc}(\mathcal{G}) \Longleftrightarrow \mathcal{L}_{u}\left(\mathcal{L}_{a}\right)=\mathcal{L}_{a}, \forall a \in G \quad \Longleftrightarrow \mathcal{L}_{\left({ }^{u} a\right)}=\mathcal{L}_{a}, \forall a \in G \\
& \Longleftrightarrow{ }^{[u]}[a]=\left[{ }^{u} a\right]=[a], \forall a \in G \Longleftrightarrow \mathcal{L}_{[u]}=i d_{G_{1}} \\
& \Longleftrightarrow \mathcal{L}_{[u]}=i d_{[X]} \quad \Longleftrightarrow \mathcal{L}_{[u]} \in \operatorname{ker} \varphi_{0} \simeq H_{0} / \mathcal{K}_{0} .
\end{aligned}
$$

For the first implication on the last line we use (5.7). By Lemma 5.4 one has $H_{0}=\mathcal{K}_{1}$ and $\operatorname{ker} \varphi_{0} \simeq \mathcal{K}_{1} / K_{0}$, hence $\operatorname{Soc}(\mathcal{G}) \simeq \mathcal{K}_{1} / \mathcal{K}_{0}$. Moreover, there are isomorphisms of symmetric groups $\mathcal{G}_{1} \simeq \mathcal{G} /\left(\operatorname{ker} \varphi_{0}\right)=\mathcal{G} / \operatorname{Soc}(\mathcal{G}) \simeq \operatorname{Ret}\left(\mathcal{G}, r_{\mathcal{G}}\right)$.

Proposition 5.7. Let $(X, r)$ be a solution.

(1) For all $j \geq 0$ there are canonical group epimorphisms

$\nu_{j}: G_{j} \longrightarrow G_{j+1}, \quad x^{(j)} \mapsto x^{(j+1)} ; \quad \mathcal{L}^{j}: G_{j} \longrightarrow \mathcal{G}_{j}, \quad x^{(j)} \mapsto \mathcal{L}_{x^{(j)}} ;$

$f_{j}: G_{j+1} \longrightarrow \mathcal{G}_{j}, \quad x^{(j+1)} \mapsto \mathcal{L}_{x^{(j)}} ; \quad \varphi_{j}: \mathcal{G}_{j} \longrightarrow \mathcal{G}_{j+1}, \quad \mathcal{L}_{x^{(j)}} \mapsto \mathcal{L}_{x^{(j+1)}}$.

(2) For $j \geq 0$ let $N_{j}$, (respectively, $\mathcal{K}_{j}, H_{j}$ ) be the pull-back in $G$ of the kernel $\operatorname{ker} \nu_{j}$, (respectively, the pull-back of $\left.\operatorname{ker} \mathcal{L}^{j}, \operatorname{ker} \varphi_{j}\right)$. These are ideals of $(G, r)$ and there are inclusions

$$
\begin{aligned}
& N_{0} \subset N_{1} \subset N_{2} \subset \cdots \subset N_{j} \subset N_{j+1} \subset \cdots \\
& \cap \cap \cap \cap \cap \\
& \mathcal{K}_{0} \subset \mathcal{K}_{1} \subset \mathcal{K}_{2} \subset \cdots \subset \mathcal{K}_{j} \subset \mathcal{K}_{j+1} \subset \cdots \\
& \|\quad\| \quad \| \\
& H_{0} \subset H_{1} \subset \cdots \subset H_{j-1} \subset H_{j} \subset \cdots
\end{aligned}
$$

The kernels satisfy

$$
\begin{aligned}
& \operatorname{ker} \nu_{j} \simeq N_{j} / N_{j-1} \quad \operatorname{ker} \mathcal{L}^{j} \simeq \mathcal{K}_{j} / N_{j-1} \\
& \operatorname{ker} f_{j} \simeq \mathcal{K}_{j} / N_{j}, \quad \operatorname{ker} \varphi_{j} \simeq \mathcal{K}_{j+1} / \mathcal{K}_{j} \simeq H_{j} / H_{j-1} \text {, } \\
& \text { where } N_{-1}:=\{1\}=: H_{-1} \text {. }
\end{aligned}
$$

(3) One has

$$
\begin{array}{ll}
G_{j} \simeq G / N_{j-1}, & \operatorname{Soc} G_{j} \simeq \mathcal{K}_{j} / N_{j-1} \\
\mathcal{G}_{j} \simeq G / \mathcal{K}_{j}, & \operatorname{Soc}\left(\mathcal{G}_{j}\right) \simeq \mathcal{K}_{j+1} / \mathcal{K}_{j} \simeq H_{j} / H_{j-1}, \forall j \geq 0 .
\end{array}
$$

So there are isomorphisms of symmetric groups:

$$
\operatorname{Ret}\left(G_{j}, r_{G_{j}}\right) \simeq\left(\mathcal{G}_{j}, r_{\mathcal{G}_{j}}\right), \quad \operatorname{Ret}\left(\mathcal{G}_{j}, r_{\mathcal{G}_{j}}\right) \simeq\left(\mathcal{G}_{j+1}, r_{\mathcal{G}_{j+1}}\right), \forall j \geq 0 .
$$


Proof. Applying Remark 5.1 to the solution $\left([X], r_{[X]}\right)$ and its symmetric group $G_{1}=G\left([X], r_{[X]}\right)$, and more generally, to $\operatorname{Ret}^{j}(X, r)$ and its symmetric group $G_{j}=G\left(\operatorname{Ret}^{j}(X, r)\right), j \geq 1$, one yields

$$
\begin{aligned}
& \mathcal{K}_{1}=\left\{u \in G \mid \mathcal{L}_{[u]}=\operatorname{id}_{[X]}\right\}=\left\{u \in G \mid \mathcal{L}_{[u]}=\operatorname{id}_{G_{1}}\right\} \\
& \mathcal{K}_{j}=\left\{u \in G \mid \mathcal{L}_{\left(u^{(j)}\right)}=\operatorname{id}_{\operatorname{Ret}^{j}(X, r)}\right\}=\left\{u \in G \mid \mathcal{L}_{\left(u^{(j)}\right)}=\operatorname{id}_{G_{j}}\right\}, j \geq 1 .
\end{aligned}
$$

A routine iteration of Lemmas 5.4 and 5.6 verifies the proposition.

Theorem 5.8. Let $(X, r)$ be a solution, notation as above.

(1) The retractions of the symmetric group $\left(G, r_{G}\right)$ satisfy

$$
\operatorname{Ret}^{j+1}\left(G, r_{G}\right) \simeq \mathcal{G}\left(\operatorname{Ret}^{j}(X, r)\right) \text {, for all } j \geq 0 \text {. }
$$

(2) The following diagram is commutative:

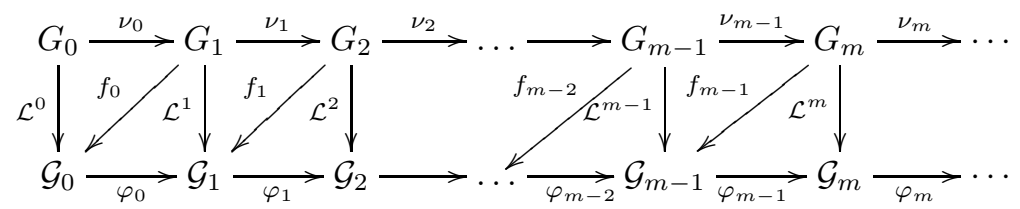

(3) The derived chain of ideals of $G$ satisfies

$$
\{1\}=K_{0} \varsubsetneqq K_{1}=\mathcal{K}_{0} \subseteq K_{2}=\mathcal{K}_{1} \subseteq \cdots \subseteq K_{m}=\mathcal{K}_{m-1} \subseteq \cdots .
$$

The following implications are in force

$$
(\operatorname{mpl} G=m+1) \Longleftrightarrow\left(\mathcal{K}_{m-1} \varsubsetneqq \mathcal{K}_{m}=G\right) \Longleftrightarrow(\operatorname{mpl} \mathcal{G}=m) .
$$

Proof. The theorem follows from Proposition 5.7

We introduce derived symmetric groups and derived permutation groups of a solution, these are in the spirit of the notion derived chain of ideals of a symmetric group.

Definition 5.9. Let $(X, r)$ be a solution. We shall call $\left(G_{j}, r_{G_{j}}\right)=G\left(\operatorname{Ret}^{j}(X, r)\right)$, $j \geq 0$, the derived symmetric groups of $(X, r)$. Similarly, the symmetric groups $\left(\mathcal{G}_{j}, r_{\mathcal{G}_{j}}\right)=\mathcal{G}\left(\operatorname{Ret}^{j}(X, r)\right), j \geq 0$, will be called the derived permutation groups of $(X, r)$. As usual $\left(G_{j},+, \cdot\right)$ and $\left(\mathcal{G}_{j},+, \cdot\right)$ denote the corresponding derived braces.

It follows from Proposition [5.7, and Theorem 5.8 that every solution $(X, r)$ has two sequences of derived symmetric groups, $\left\{\left(G_{j}, r_{G_{j}}\right) \mid j \geq 0\right\}$ and $\left\{\left(\mathcal{G}_{j}, r_{\mathcal{G}_{j}}\right) \mid j \geq 0\right\}$. Each of these sequences is an invariant of the solution and reflects the process of retraction. The sequences are closely related and satisfy

$$
\left(\mathcal{G}_{j}, r_{\mathcal{G}_{j}}\right) \simeq \operatorname{Ret}\left(G_{j}, r_{G_{j}}\right), \operatorname{Ret}\left(\mathcal{G}_{j}, r_{\mathcal{G}_{j}}\right) \simeq\left(\mathcal{G}_{j+1}, r_{\mathcal{G}_{j+1}}\right), \operatorname{Ret}^{j+1}\left(G, r_{G}\right) \simeq\left(\mathcal{G}_{j}, r_{\mathcal{G}_{j}}\right) .
$$

In general, each of the derived sequences may have repeating members, see Example 5.13. In the special case when $(X, r)$ is non-retractable one has $\left(G_{j}, r_{G_{j}}\right)=\left(G, r_{G}\right)$, and $\left(\mathcal{G}_{j}, r_{\mathcal{G}_{j}}\right)=\left(\mathcal{G}, r_{\mathcal{G}}\right)$, for all $j \geq 0$. Each derived symmetric group and each derived permutation group encodes various combinatorial properties of the solution $(X, r)$ and may have strong impact on it. This is illustrated by Corollary 5.16. (2), Theorem 8.2, and Corollary 8.7 . 
Corollary 5.10. If the groups $\mathcal{G}(X, r)$ and $\mathcal{G}_{1}(X, r)$ are not isomorphic, then $(X, r) \neq \operatorname{Ret}(X, r)$ and therefore the solution $(X, r)$ is retractable.

Remark 5.11. There are numerous examples of solutions $(X, r)$ such that $\operatorname{Ret}(X, r) \neq$ $(X, r)$, but $\mathcal{G}=\mathcal{G}(X, r) \simeq \mathcal{G}(\operatorname{Ret}(X, r))=\mathcal{G}_{1}$. In fact any trivial extension $(X, r)=Y \bigcup Z$ of an irretractable solution $\left(Y, r_{Y}\right),|Y| \geq 2$, with a trivial solution $\left(Z, r_{Z}\right)$, where $|Z| \geq 2$, satisfies $(X, r) \neq \operatorname{Ret}(X, r)$ but $\mathcal{G}(X, r) \simeq \mathcal{G}(\operatorname{Ret}(X, r))$. Example 5.13 presents a concrete solutions with these properties.

The unique non-retractable square-free solution $\left(Y, r_{Y}\right)$ of order 8 was noticed first by Vendramin in the complete list of solutions of order 8 presented by Schedler (who used a computer programme). Vendramin wrote: "It is remarkable that this is the only counterexample to the Gateva-Ivanova's conjecture among the 2471 square-free solutions of size $|X|=8$ !". We recall its definition below.

Remark 5.12. Let $\left(Y, r_{Y}\right)$ be the square-free solution of order 8 given in $\mathrm{Ve}$ Example 3.9. It is defined as

$$
Y=\left\{x_{1}, x_{2}, x_{3}, x_{4}, x_{5}, x_{6}, x_{7}, x_{8}\right\}, \quad r(x, y)=\left(\mathcal{L}_{x}(y), \mathcal{L}_{y}^{-1}(x)\right),
$$

$\mathcal{L}_{x_{1}}=\left(x_{5} x_{7}\right), \mathcal{L}_{x_{3}}=\left(x_{5} x_{7}\right)\left(x_{2} x_{6}\right)\left(x_{4} x_{8}\right), \mathcal{L}_{x_{5}}=\left(x_{1} x_{3}\right), \mathcal{L}_{x_{7}}=\left(x_{1} x_{3}\right)\left(x_{2} x_{6}\right)\left(x_{4} x_{8}\right)$,

$\mathcal{L}_{x_{2}}=\left(x_{6} x_{8}\right), \mathcal{L}_{x_{4}}=\left(x_{6} x_{8}\right)\left(x_{1} x_{5}\right)\left(x_{3} x_{7}\right), \mathcal{L}_{x_{6}}=\left(x_{2} x_{4}\right), \mathcal{L}_{x_{8}}=\left(x_{2} x_{4}\right)\left(x_{1} x_{5}\right)\left(x_{3} x_{7}\right)$.

Clearly, the solution $\left(Y, r_{Y}\right)$ is irretractable. One has $\mathcal{G}=\mathcal{G}\left(Y, r_{Y} \simeq D_{4}\right.$, the dihedral group of 8 elements, see $\mathrm{Ve}$. The set $Y$ splits into two $\mathcal{G}$-orbits: $Y_{1}=$ $\left\{x_{1}, x_{3}, x_{5}, x_{7}\right\}$ and $Y_{2}=\left\{x_{2}, x_{4}, x_{6}, x_{8}\right\}$, denote the induced solution by $\left(Y_{1}, r_{1}\right)$, $\left(Y_{2}, r_{2}\right)$. Then $\operatorname{mpl}\left(Y_{i}, r_{i}\right)=2, i=1,2$. It is easy to see that $\left(Y, r_{Y}\right)=Y_{1} \natural Y_{2}$ is a strong twisted union of solutions of multipermutation level 2 .

Therefore $\left(Y, r_{Y}\right)$ provided the first negative answer to a question posed by the author and Cameron: "Is it true that a strong twisted union of two multipermutation solutions is also a multipermutation solution?", GIC12, Open Questions 6.13.

Example 5.13. Let $X=Y \natural_{0} Z$ be the trivial strong twisted union of the solutions $\left(Y, r_{Y}\right)$ and $\left(Z, r_{Z}\right)$, where $\left(Y, r_{Y}\right)$ is the irretractable solution (5.11), and $\left(Z, r_{Z}\right)$ is the trivial solution on the two-elements set $Z=\{a, b\}$. By definition $X=Y \bigcup Z$ and the map $r$ is defined as $r(u, v)=\left(\mathcal{L}_{u}(v), \mathcal{L}_{v}^{-1}(u)\right), u, v \in X$, where $\mathcal{L}_{a}=\mathcal{L}_{b}=$ $i d_{X}$, and the maps $\mathcal{L}_{y}, y \in Y$, are given in (5.11). Thus $\mathcal{L}_{y} \neq \mathcal{L}_{v}$, whenever $y \in$ $Y, v \in X, v \neq y$. The permutation group $\mathcal{G}(X, r)$ is a subgroup of $\operatorname{Sym}(X)=S_{10}$ and is generated by $\mathcal{L}_{u}, u \in X$. In fact, each map $\mathcal{L}_{y}$ considered as permutation of $X$ moves only elements of $Y$, so $\left(\mathcal{L}_{y}\right)_{\mid Y}=\mathcal{L}_{y}$, and the set $\mathcal{L}_{y}, y \in Y$ generates the whole group $\mathcal{G}(X, r)$. Therefore $\mathcal{G}(X, r) \simeq \mathcal{G}\left(Y, r_{Y}\right) \simeq D_{4}$. It is clear that $[a]=[b]$ in the retraction $\left([X], r_{[X]}\right)$, and $[y] \neq[v]$, whenever $y \in Y, v \in X, y \neq v$, hence the retraction $\left([X], r_{[X]}\right)=\operatorname{Ret}(X, r)$ is a solution with cardinality 9 . More precisely, $\operatorname{Ret}(X, r)=Y \natural_{0}\{[a]\}$, is a strong twisted union of $Y$ with a one element solution. Obviously, $(X, r)$ is not isomorphic to $\operatorname{Ret}(X, r)$, but $\mathcal{G}_{1}=\mathcal{G}(\operatorname{Ret}(X, r)) \simeq \mathcal{G} \simeq D_{4}$ and the socle $\operatorname{Soc}(\mathcal{G})=\{1\}$ is trivial. In contrast the groups $G=G(X, r)$ and $G_{1}=G(\operatorname{Ret}(X, r))$ are not isomorphic. Moreover $\operatorname{Ret}^{2}(X, r)=\operatorname{Ret}^{1}(X, r)$, so $\operatorname{Ret}^{1}(X, r)$ is non-retractable and the process of retraction of $(X, r)$ halts after the first step. The derived chain of ideals satisfies $1 \neq K_{0}=K_{1} \varsubsetneqq G$.

It is known that the minimal order of a non-retractable solution $(X, r)$ is $|X|=4$. 
Lemma 5.14. Assumptions and notation as above. The diagram (5.9) is finite iff there exists an integer $m \geq 0$, such that $\operatorname{Ret}^{m}(X, r)=\operatorname{Ret}^{m+1}(X, r)$. Suppose this is the case, and let $m$ be the minimal integer with this property. Exactly one of the following two conditions is satisfied:

(1) The retraction $\operatorname{Ret}^{m}(X, r)$ is a one element solution, so mpl $X=m \geq 1$. Then $m \leq \operatorname{mpl} G(X, r) \leq m+1$, and the diagram has the shape

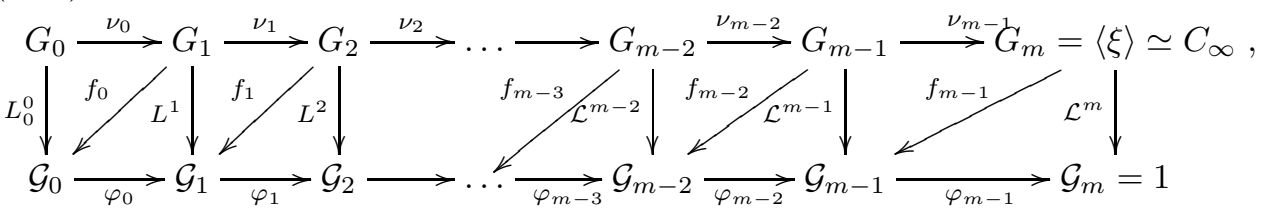

where $\xi=x^{(m)}$ is the $m$-th retraction of an arbitrary $x \in X$, and $\mathcal{G}_{m-1}$ is a finite cyclic group $\left(\mathcal{G}_{m-1}=1\right.$ is possible).

(2) $\operatorname{Ret}^{m}(X, r)$ is a non-retractable solution of order $n \geq 4, G_{m}=G_{m+1}$ is an $n$-generated torsion-free group. Moreover $\mathcal{G}_{m}=\mathcal{G}_{m+1}$ is a nonabelian finite group with $\operatorname{Soc} \mathcal{G}_{m}=\{1\}$.

Theorem 5.15. Let $(X, r)$ be a non-degenerate symmetric set of order $|X| \geq 2$ (not necessarily finite), notation as above.

(1) The symmetric group $\left(G, r_{G}\right)$ has finite multipermutation level iff $(X, r)$ is a multipermutation solution. In this case one has

$$
0 \leq \operatorname{mpl}\left(\mathcal{G}, r_{\mathcal{G}}\right)=m-1 \leq \operatorname{mpl}(X, r) \leq \operatorname{mpl}\left(G, r_{G}\right)=m<\infty .
$$

(2) Suppose furthermore that $(X, r)$ satisfies condition $(*)$. Then

$$
\operatorname{mpl} X=m<\infty \text { iff } \operatorname{mpl} G=m<\infty .
$$

In particular, (5.14) is in force for square-free solutions $(X, r)$ of arbitrary cardinality.

Proof. (1). Consider $\left(G, r_{G}\right)$ as a symmetric set. Then $X$ is an $r_{G}$-invariant subset of $G$. Suppose $\left(G, r_{G}\right)$ has a finite multipermutation level $\operatorname{mpl} G=m<\infty$. Note that the left action of $G$ upon itself extends canonically the left action of $G$ upon $X$. Moreover, the equality $K=\operatorname{Soc} G=\Gamma$ implies that two elements $x, y \in X$ have the same action $\mathcal{L}_{x}=\mathcal{L}_{y}$ on $X$ if and only if they have the same action on $G$. Our assumption $\operatorname{mpl} G=m$ implies that (4.5) is in force for every choice of $x, y_{1}, \cdots, y_{m} \in G$. But $X$ is embedded in $G$, hence, (4.5) is satisfied by any $x, y_{1}, \cdots, y_{m} \in X$, and therefore by Proposition $4.7 \mathrm{mpl} X \leq m$. This verifies the implication

$$
\operatorname{mpl} G=m<\infty \Longrightarrow 1 \leq \operatorname{mpl} X \leq \operatorname{mpl} G \text {. }
$$

Assume now that $(X, r)$ is a multipermutation solution of level $m=\operatorname{mpl} X$. Then, by Lemma $5.14 \mathcal{G}_{m}=\{1\}$ is a one element solution. But $\mathcal{G}_{m} \simeq \operatorname{Ret}^{m+1}\left(G, r_{G}\right)$, hence $\operatorname{mpl} G \leq m+1$. More precisely, either (a) $\mathcal{G}_{m-1}$ is a nontrivial finite cyclic group, then $\operatorname{mpl} G=m+1$, and $\operatorname{mpl} \mathcal{G}=\operatorname{mpl} X=m$; or (b) $\mathcal{G}_{m-1}=\{1\}$, then $\operatorname{Ret}^{m}\left(G, r_{G}\right)$ is a one element solution ( $m$ is minimal with this property). In this case $\operatorname{mpl} X=m=\operatorname{mpl} G$, and $\operatorname{mpl}\left(\mathcal{G}, r_{\mathcal{G}}\right)=m-1$. (2). Suppose $(X, r)$ 
satisfies condition $\left(^{*}\right)$, and $\mathrm{mpl} X=m$. Then $\operatorname{Ret}^{m-1}(X, r)$ is a trivial solution of order $\geq 2, G_{m-1}$ is a free abelian group of finite rank, hence $\mathcal{G}_{m-1}=\{1\}$. By Theorem $5.8 \operatorname{Ret}^{m}\left(G, r_{G}\right) \cong \mathcal{G}_{m-1}$ so it is a one element solution, and if $m \geq 2$, $\operatorname{Ret}^{m-1}\left(G, r_{G}\right)=\mathcal{G}_{m-2}$ has order $\geq 2$. Therefore $\operatorname{mpl} G=m=\operatorname{mpl} X$.

Corollary 5.16. Let $(X, r)$ be a finite solution, notation as usual.

(1) Suppose the left brace $(\mathcal{G},+, \cdot)$ is a nontrivial two-sided brace.

(a) The corresponding radical ring $\mathcal{G}^{\star}$ is nilpotent, say of class $m>1$;

(b) In this case $(X, r)$ is a multipermutation solution, with $m-1 \leq$ $\operatorname{mpl} X \leq m$, and the inequalities (5.13) are in force. Moreover, if $(X, r)$ is a square free-solution, then

$$
\operatorname{mpl}(X, r)=m=\text { the nilpotency degree of } \mathcal{G}^{\star} .
$$

(2) $(X, r)$ is a multipermutation solution with $\mathrm{mpl} X \geq 2$ iff there exist some $j \geq 0$ such that $\left(\mathcal{G}_{j},+, \cdot\right)$ is a two-sided brace of order $>1$.

Proof. (1). It is well-known that the Jacobson radical of a left Artinian ring is nilpotent, see for example Lam, Theor. 4.12. This straightforwardly implies that a finite radical ring $R$ is nilpotent, so (1a) holds. By Theorem $4.21 \mathrm{mpl}\left(\mathcal{G}, r_{\mathcal{G}}\right)=m-1$ so $\operatorname{mpl}\left(G, r_{G}\right)=m$. Now part (1b) follows straightforwardly from Theorem 5.13 ,

(2). Assume $\left(\mathcal{G}_{j},+, \cdot\right)$ is a two-sided brace for some $j \geq 0,\left|\mathcal{G}_{j}\right|>1$. Part (1) implies that $\operatorname{Ret}^{j}(X, r)$ is a multipermutation solution, say $\operatorname{mpl} \operatorname{Ret}^{j}(X, r)=s$, hence $\operatorname{mpl}(X, r)=s+j<\infty$. Conversely, suppose $\operatorname{mpl} X=m \geq 2$. Then $m-1 \leq$ $s=\operatorname{mpl} \mathcal{G} \leq m$, so $\operatorname{mpl} \mathcal{G}_{s-1}=1$, and therefore, by Remark 4.19 $\mathcal{G}_{s-1}$ is a two-sided brace.

\section{A symmetric Set $(X, r)$ Whose Brace $G(X, r)$ is tWo-Sided, or SQUARE-FREE MUST BE A TRIVIAL SOLUTION}

In this section $(X, r)$ denotes (a non-degenerate) symmetric set, not necessarily finite. As usual, $S=S(X, r)$ is the associated YB monoid, $\left(G, r_{G}\right)$ and $(G,+, \cdot)$, are the associated symmetric group and left brace, respectively, where $G=G(X, r)$. We shall show that each of the conditions: " $G$ is a two-sided brace"; or " $\left(G, r_{G}\right)$ is a square-free solution", is extremely restrictive. The main result of the section is the following theorem.

Theorem 6.1. Suppose $(X, r)$ is a non-degenerate symmetric set (not necessarily finite). The following conditions are equivalent.

(1) The associated left brace $(G,+, \cdot)$ is a two-sided brace.

(2) The associated symmetric group $\left(G, r_{G}\right)$ is a square-free solution.

(3) $(X, r)$ is a square-free solution and ${ }^{x y}(x y)=x y, \forall x, y \in G$, holds in $G$.

(4) The multiplicative group $G=G(X, r)$ is a free abelian group generated by $X$, so $\left(G, r_{G}\right)$ is the trivial solution.

(5) $(X, r)$ is a trivial (square-free) solution.

The implication $(2) \Longrightarrow(1)$ was proven independently in [CJO14, Theorem 5]. Our proof of Theorem 6.1 does not use their result. We apply our "symmetric groups 
-braces" approach to show a stronger statement: each of the conditions (1) and (2) is equivalent to " $(X, r)$ is a trivial solution". We prove first some more results.

Lemma 6.2. Let $(G,+, \cdot)$ be a left brace, and let $(G, r)$ be the corresponding symmetric group. (1) $G$ is a two-sided brace if and only if

$$
c\left(\left(^{(a b c)^{-1}} c\right)=\left({ }^{b^{-1}} c\right)\left(\left(\left(a^{b}\right)\left(^{b^{-1}} c\right)\right)^{-1} c\right), \quad \forall a, b, c \in G .\right.
$$

(2) Assume that $(G, r)$ satisfies lri. Then $G$ is a two-sided brace if and only if

$$
c\left(c^{(a b c)}\right)=\left(c^{b}\right)\left(c^{\left(a^{b} c^{b}\right)}\right), \quad \forall a, b, c \in G .
$$

The proof of Lemma 6.2 involves technical computations in the symmetric group $(G, r)$ and can be found in the first two versions of our preprint, see arXiv:1507.02602 [math.QA]. The original proof of Theorem 6.3 can be also found in the first two versions of the same preprint. Here we give a second proof whose idea was kindly suggested to the author by Ferran Cedó.

Theorem 6.3. Let $(X, r)$ be a symmetric set (not necessarily finite), and let $(G,+, \cdot)$, be the associated left brace. If $(G,+, \cdot)$ is a two-sided brace then $(X, r)$ is the trivial solution on the set $X$.

Proof. Assume $(G,+, \cdot)$ is a two-sided brace and consider the associated Jacobson radical ring $G^{*}=(G,+, *)$, where $a * b:=a b-a-b$ or equivalently, $a b=a * b+a+b$. In particular, a left, and a right distributive law hold in $G^{\star}$. Moreover, by (4.10), one has ${ }^{a} b=a * b+b, \forall a, b \in G$. Let $x, y \in X$, then one has:

$$
{ }^{(x+x)} y=(x+x) * y+y=x * y+x * y+y=2(x * y+y)-y=2\left({ }^{x} y\right)-y .
$$

So the following identity is in force in the additive group $(G,+)$ :

$$
y+{ }^{(x+x)} y-2\left({ }^{x} y\right)=0, \forall x, y \in X .
$$

Note first that $y,{ }^{(x+x)} y,{ }^{x} y \in X$. Recall that the set $X$ is embedded in $G$ and the additive group $(G,+)$ is isomorphic to the (additive) free abelian group, ${ }_{g r}[X]$ generated by $X$, see Remark [3.9. Then (6.3) must be a trivial relation, which is possible if and only if ${ }^{x} y=y={ }^{(x+x)} y$. This proves that ${ }^{x} y=y$, for all $x, y \in X$, and therefore $(X, r)$ is a trivial solution.

Proof of Theorem. 6.1. The implications (5) $\Longleftrightarrow$ (4) $\Longrightarrow(10),(5) \Longrightarrow(2) \Longrightarrow(3)$ are obvious. Theorem 6.3 verifies the implication (10) $\Longrightarrow$ (5).

(3) $\Longrightarrow$ (5). Let $x, y \in X, x \neq y$. Then $x y={ }^{x y}(x y)=\left({ }^{x y} x\right)\left(\left({ }^{x y}\right)^{x} y\right)$, by ML2. Therefore the following equality of monomials of length two holds in the monoid $S=S(X, r)$ :

$$
x y=\left({ }^{x y} x\right)\left({ }^{(x y)^{x}} y\right) .
$$

Two cases are possible. 1. The equality (6.4) holds in the free monoid $\langle X\rangle$. In this case ${ }^{(x y)} x=x$. Moreover, ${ }^{(x y)} x={ }^{x}\left({ }^{y} x\right)={ }^{x} x$, which by the non-degeneracy of $(X, r)$ implies ${ }^{y} x=x$. 2. (6.4) is not an equality of words in $\langle X\rangle$. Then it is a (non-trivial) relation for $S$, therefore $\left({ }^{x} y, x^{y}\right)=r(x, y)=\left({ }^{x y} x,\left({ }^{x} y\right)^{x} y\right)$ holds in $X \times X$. Hence ${ }^{x} y={ }^{x y} x={ }^{x}\left({ }^{y} x\right)$. The non-degeneracy again implies $y={ }^{y} x$. But $(X, r)$ is a square-free solution, so $y={ }^{y} y$, and ${ }^{y} x=y={ }^{y} y$ imply $x=y$, which contradicts our assumption $x, y \in X, x \neq y$. Therefore case $\mathbf{2}$ is impossible. 
It follows that the equality ${ }^{y} x=x$ holds for all $x, y \in X$, that is $\mathcal{L}_{y}=i d_{X}$, for all $y \in X$, hence $(X, r)$ is a trivial solution.

\section{Symmetric GROUPS With CONDITIONS lri AND Raut}

In this section we introduce some special conditions on the actions of a symmetric group $(G, r)$ upon itself and study their effect on the properties of $(G, r)$ and the associated brace $(G,+, \cdot)$.

7.1. Symmetric sets $(X, r)$ with condition lri. We present first some useful technical results. As usual, $(X, r)$ denotes a non-degenerate symmetric set, $\left(G, r_{G}\right)$, and $(G,+, \cdot)$ denote its associated symmetric group and left brace, respectively.

Recall that condition lri holds on $(X, r)$ iff $(X, r)$ satisfies the cyclic conditions cc, see Corollary 2.8. Moreover, every square-free solution satisfies lri. The permutation solutions, see Example 4.1, are a well known class of solutions with condition lri. Recently, an interesting class of non-retractable solutions each of which satisfies lri but is not necessarily square-free were introduced and studied in BCJO]. Proposition 7.13 shows that every symmetric group $(G, r)$ with $\operatorname{mpl} G=2$ satisfies lri and conditions (7.1) given below. Note that if $(X, r)$ is with lri, then its symmetric group $\left(G, r_{G}\right)$ is a symmetric set which, in general, may not satisfy condition lri. However, a mild generalization of lri denoted by lri $\star$ is always in force, see Proposition 7.6. The special cases when some of the symmetric group $\left(G, r_{G}\right)$, or $\left(\mathcal{G}, r_{\mathcal{G}}\right)$ satisfies lri will be studied in the next section.

Lemma 7.1. Let $(X, r)$ be a nontrivial symmetric set of arbitrary cardinality, $|X| \geq 2$. The following two conditions are equivalent.

$$
\text { (i) } \quad \mathcal{L}_{x_{a}}=\mathcal{L}_{a}, \quad \forall a, x \in X ; \quad \text { (ii) } \quad \mathcal{L}_{a^{x}}=\mathcal{L}_{a}, \quad \forall a, x \in X .
$$

Moreover, each of these conditions implies condition lri.

Proof. (i) $\Longrightarrow$ (ii) and lri. Assume (i) is in force, and let $a, x \in X$. Applying first (2.1) and then (7.1) (i) we obtain $x={ }^{x} a\left(x^{a}\right)={ }^{a}\left(x^{a}\right)$. This gives the first lri identity ${ }^{x}\left(a^{x}\right)=a, \forall a, x \in X$ which together with (7.1) (i) imply $\left.\mathcal{L}_{a}=\mathcal{L}_{(x}\left(a^{x}\right)\right)=$ $\mathcal{L}_{a^{x}}, \forall a, x \in X$, hence (ii) is in force. We use (2.1) and (ii) to yield

$$
x=\left({ }^{a} x\right)^{\left(a^{x}\right)}=\left({ }^{\left(a^{x}\right)} x\right)^{\left(a^{x}\right)},
$$

hence (by the non-degeneracy) the second lri identity $\left({ }^{a} x\right)^{a}=x, \quad \forall a, x \in X$, is also in force. Analogous argument verifies the implication (ii) $\Longrightarrow$ (i) and lri.

Proposition 7.2. Let $(X, r)$ be a non-degenerate symmetric set, with associated symmetric group $G=G(X, r)$. Let $X_{i}, i \in I$, be the set of all $G$-orbits in $X$ (possibly infinitely many). Suppose that for each $i \in I,\left(X_{i}, r_{i}\right)$ is a trivial solution, or one element solution. Then

(1) The following equalities hold for all $y \in X, a, b \in X_{i}, i \in I$ :

$$
{ }^{b} y={ }^{y} a, \quad y^{b} a={ }^{y} a, \quad a^{y^{b}}=a^{y}, \quad a^{b} y=a^{y} .
$$

(2) $X$ is a strong twisted union $X=\bigsqcup_{i \in I} X_{i}$, in the sense of [GIC12].

(3) $(X, r)$ satisfies condition lri. 
Proof. (1). By hypothesis each orbit is a trivial or one element solution, therefore

$$
{ }^{b} a=a=a^{b}, \quad \forall a, b \in X_{i} .
$$

Assume now $a, b \in X_{i}, i \in I$, and $y \in X$. Then $a, b,{ }^{y} a, b^{y} \in X_{i}$ so we apply (17.3) twice to deduce ${ }^{y} a={ }^{b}\left({ }^{y} a\right)={ }^{b} y\left(b^{y} a\right)={ }^{b} y a$. This verifies the first equality in (7.2). The proof of the remaining three equalities is analogous. (2). Clearly, $X$ is a disjoint union $X=\bigcup_{i \in I} X_{i}$ of its $G$-orbits. It follows from (7.2) that for each pair $i, j \in I, i \neq j, X_{i} \llbracket X_{j}$ is a strong twisted union, but this means that $X$ is a strong twisted union $X=\natural_{i \in I} X_{i}$, in the sense of GIC12. (3). We shall prove that lri holds. Let $a, x \in X$, say $x \in X_{i}, i \in I$. Then $x, x^{a} \in X_{i}$, so (2.1) and (7.2) imply $x={ }^{x} a\left(x^{a}\right)={ }^{a}\left(x^{a}\right)$, which gives the first lri equality, $x={ }^{a}\left(x^{a}\right)$. The second equality, $\left({ }^{a} x\right)^{a}=x$, is proven analogously.

Remark 7.3. If $(X, r)$ is a symmetric set with lri then

$$
c\left(b^{c}\right)=b\left(c^{b}\right), \quad \text { for all } b, c \in X .
$$

Indeed, conditions M3, lri and the cyclic condition cc imply: $c\left(b^{c}\right)=\left({ }^{c}\left(b^{c}\right)\right) c^{b^{c}}=$ $b\left(c^{b}\right)$.

Notation 7.4. $\quad X^{\star}:=X \bigcup X^{-1}, \quad$ where $\quad X^{-1}=\left\{x^{-1} \mid x \in X\right\}$.

Lemma 7.5. Let $(X, r)$ be a symmetric set, $G=G(X, r)$ in the usual notation.

(1) The following implications are in force for all $a, b \in G$.

$$
\left(a^{-1} b=b^{a}\right) \Longleftrightarrow\left({ }^{a}\left(b^{a}\right)=b\right) ; \quad\left(b^{a^{-1}}={ }^{a} b\right) \Longleftrightarrow\left(\left({ }^{a} b\right)^{a}=b\right) .
$$

(2) The following equalities hold for all $a, b \in G$.

$$
\begin{gathered}
a^{\left(a^{-1} b\right)}={ }^{b^{-1}} a, \quad\left(a^{-1}\right)^{b}=\left(a^{\left(a^{-1} b\right)}\right)^{-1}, \quad{ }^{b}\left(a^{-1}\right)=\left({ }^{\left(b^{a^{-1}}\right)} a\right)^{-1}, \\
\left({ }^{b} a\right)^{-1}=\left(a^{-1}\right)^{\left(b^{-1}\right)}, \quad\left({ }^{b}\left(a^{-1}\right)\right)^{-1}=a^{\left(b^{-1}\right)}, \quad\left({ }^{b^{-1}} a\right)^{-1}=\left(a^{-1}\right)^{b}, \quad\left(b^{-1} a^{-1}\right)^{-1}=a^{b} .
\end{gathered}
$$

The proof of this lemma is given in GI15, p.18.

The condition lri on $(X, r)$ can be extended for the actions of the whole group $G$ upon $X^{\star}$, as shows the following result.

Proposition 7.6. In notation as above, let $(X, r)$ be a symmetric set with lri, and let $G=G(X, r)$ be its $Y B$ group.

(1) The following generalization of lri holds:

$$
\begin{gathered}
\operatorname{lri} \star: \quad{ }^{a}\left(x^{a}\right)=x=\left({ }^{a} x\right)^{a}, \quad \forall x \in X^{\star}, a \in G . \\
{ }^{a^{-1}} x=x^{a}, \quad x^{a^{-1}}={ }^{a} x, \quad \forall x \in X^{\star}, a \in G .
\end{gathered}
$$

(2) The following equalities hold:

$$
\left(x^{a}\right)^{-1}=\left(x^{-1}\right)^{a}, \quad\left({ }^{a} x\right)^{-1}={ }^{a}\left(x^{-1}\right), \quad \forall x \in X^{\star}, a \in G .
$$


(3) The following generalization of the cyclic conditions is in force.

$$
\operatorname{cl1} \star: \quad a^{x} x={ }^{a} x, \quad \operatorname{cr} 1 \star: \quad x^{x} a=x^{a}, \quad \forall x \in X^{\star}, a \in G .
$$

More generally,

(4) The following equalities hold.

$$
{ }^{a}\left(x^{k}\right)=\left({ }^{a} x\right)^{k}, \quad\left(x^{k}\right)^{a}=\left(x^{a}\right)^{k} \quad \forall k \in \mathbb{N}, x \in X^{\star}, a \in G .
$$

A detailed proof of the proposition can be found in GI15.

Corollary 7.7. Let $(X, r)$ be a symmetric set with lri. (1) The set $X^{\star}$ is $r_{G^{-}}$ invariant. (2) Let $r^{\star}$ be the restriction of $r_{G}$ on $X^{\star} \times X^{\star}$. Then $\left(X^{\star}, r^{\star}\right)$ is a symmetric set which satisfies lri and the cyclic conditions $\boldsymbol{c c}$.

Remark 7.8. In assumption and notation as in Proposition [7.6 the following equality is in force for all $a, x, y \in X^{\star}$ :

$$
x^{a}+\left({ }^{x} y\right)^{a}={ }^{a^{-1}}(x y) .
$$

Proof. Suppose $a, x, y \in X^{\star}$, then the following equalities hold in $G$ :

$$
\begin{aligned}
x^{a}+\left({ }^{x} y\right)^{a} & ={ }^{a^{-1}}(x)+{ }^{a^{-1}}\left({ }^{x} y\right) & & \text { by lri } \star \\
& ={ }^{a^{-1}}\left(x+{ }^{x} y\right)={ }^{a^{-1}}(x y) & & \text { by Laut. }
\end{aligned}
$$

7.2. Symmetric groups with conditions lri and Raut. In this subsection, as usual, $(G, r)$ is a symmetric group and $(G,+, \cdot)$ is the associated left brace.

Definition 7.9. (1) The symmetric group $G$ acts upon itself as automorphisms if condition MLaut given below is in force:

$$
\text { MLaut: } \quad{ }^{a}(b c)=\left({ }^{a} b\right)\left({ }^{a} c\right), \quad \forall a, b, c \in G .
$$

(2) The brace $(G,+, \cdot)$ satisfies condition Raut if

$$
\text { Raut: } \quad(a+b)^{c}=a^{c}+b^{c}, \quad \forall a, b, c \in G .
$$

In this case the group $(G, \cdot)$ acts upon $(G,+)$ from the right as automorphisms. The map $\mathcal{R}:(G, \cdot) \longrightarrow \operatorname{Aut}(G,+)$ is a homomorphism of groups.

We have shown in Proposition 3.5 that under the assumption that $(G,+, \cdot)$ is a nonempty set with operations "." and "+" such that $(G, \cdot)$ is a group, $(G,+)$ is an abelian group, and the map $G \times G \longrightarrow G$ is defined as $(a, b) \mapsto{ }^{a} b:=a b-a$, condition Laut, see (3.9), is equivalent to the property " $(G,+, \cdot)$ is a left brace". In contrast, condition Raut which seems to be "a right analogue" of Laut, does not imply that the left brace $(G,+, \cdot)$ is also a right brace. The difference is due to the 'asymmetric' definitions of the left and the right actions, see (3.18).

Suppose $(G, r)$ is a symmetric group and ${ }^{a} a=a, \forall a \in G$, i.e., $(G, r)$ is a square-free solution. Then (i) $(G, r)$ satisfies conditions lri, cc and Raut; (ii) The corresponding left brace $(G,+, \cdot)$ is a two-sided brace, see CJO14, Theorem 5 .

Theorem 7.10. Let $(G, r)$ be a symmetric group and let $(G,+, \cdot)$ be the associated left brace. Then the following conditions hold. 
(1) If $(G, r)$ satisfies condition lri then condition Raut holds in $(G,+, \cdot)$. Moreover the following equality is in force

$$
\left({ }^{\left({ }^{v} a\right)} u\right)\left({ }^{a} v\right)=\left({ }^{a} u\right)\left({ }^{\left(a^{u}\right)} v\right), \quad \forall a, u, v \in G .
$$

(2) Suppose that $G=G\left(X, r_{0}\right)$ is the symmetric group of a solution $\left(X, r_{0}\right)$. If $\left(X, r_{0}\right)$ satisfies lri and condition Raut holds in $(G,+, \cdot)$, then $(G, r)$ satisfies condition lri.

Proof. (1). Suppose $a, u, v \in G$. We apply successively lri for the first, Laut, for the second, and lri for the third equality below and yield:

$$
(u+v)^{a}={ }^{a^{-1}}(u+v)={ }^{a^{-1}} u+{ }^{a^{-1}} v=u^{a}+v^{a} .
$$

Hence $(u+v)^{a}=u^{a}+v^{a}$, for all $a, u, v \in G$, that is Raut is in force.

We shall verify (7.16). Suppose $a, u, v \in G$. We apply successively MR2, Lemma 7.5 and lri and obtain:

$$
(u v)^{a^{-1}}=\left(u^{\left(v\left(a^{-1}\right)\right)}\right)\left(v^{a^{-1}}\right)=\left(u^{\left({ }^{v} a\right)^{-1}}\right)\left(v^{a^{-1}}\right)=\left({ }^{(v a)} u\right)\left({ }^{a} v\right) .
$$

Applying lri and ML2 we get $(u v)^{a^{-1}}={ }^{a}(u v)=\left({ }^{a} u\right)\left({ }^{\left(a^{u}\right)} v\right)$, which together with (7.17) implies (7.16).

(2) Assume now that $\left(X_{0}, r_{0}\right)$ is a solution with lri, and its associated left brace $(G,+, \cdot)$ satisfies Raut. We have to show that the symmetric group $(G, r)$ satisfies lri. First we use induction on the length $|a|$ of $a$ to prove that

$$
a^{\left(x^{-1}\right)}={ }^{x} a, \quad{ }^{\left(x^{-1}\right)} a=a^{x}, \quad \text { for all } a \in G, x \in X^{\star} .
$$

By hypothesis $(X, r)$ satisfies lri, hence, by Proposition 7.6, condition lri $\star$ is also in force. Therefore (7.18) is satisfied for all $a \in G,|a|=1$, which gives the base for induction. Assume (7.18) is true for all $a \in G,|a| \leq n$. Let $x \in X^{\star}$, and let $b \in G,|b|=n+1$. Then $b=a t=a+{ }^{a} t$, where $a \in G,|a|=n, t \in X^{\star}$. One has

$$
\begin{aligned}
b^{\left(x^{-1}\right)}=(a t)^{\left(x^{-1}\right)} & =\left(a+{ }^{a} t\right)^{\left(x^{-1}\right)}=a^{\left(x^{-1}\right)}+\left({ }^{a} t\right)^{\left(x^{-1}\right)} & & \text { by Raut } \\
& ={ }^{x} a+{ }^{x}\left({ }^{a} t\right)={ }^{x}\left(a+{ }^{a} t\right)={ }^{x}(a t)={ }^{x} b & & \text { by IH, and by Laut, }
\end{aligned}
$$

which proves the first equality in (7.18). The second equality in (7.18) follows straightforwardly. Next, using induction on the length $|u|$ of $u \in G$ we verify

$$
\left({ }^{u} a\right)^{u}=a,{ }^{u}\left(a^{u}\right)=a, \quad \forall a, u \in G .
$$

The base for induction follows from (7.18). Assume that (7.19) is true for all $a \in G$ and all $u \in G,|u| \leq n$. Let $a \in G$, and $v \in G$, with $|v|=n+1$. Then $v=u x$, where $u \in G,|u|=n, x \in X^{\star}$. In the following computation we use ML1, and MR1 for the second equality, and the inductive hypothesis for the third and fourth equalities:

$$
\left({ }^{v} a\right)^{v}=\left({ }^{u x} a\right)^{u x}=\left(\left({ }^{u}\left({ }^{x} a\right)\right)^{u}\right)^{x}=\left({ }^{x} a\right)^{x}=a .
$$

This proves the first identity in (7.19), the second one is verified analogously. Hence $(G, r)$ satisfies lri.

Corollary 7.11. Suppose $(X, r)$ is a solution with $\mathbf{l r i},\left(G, r_{G}\right)$ and $(G,+, \cdot)$ are its symmetric group and its left brace, respectively. Then $\left(G, r_{G}\right)$ satisfies lri if and only if $(G,+, \cdot)$ satisfies Raut. 
7.3. A symmetric group acting upon itself as automorphisms is a multipermutation solution of level $\leq 2$.

Lemma 7.12. Let $(G, r)$ be a symmetric group. The following four conditions are equivalent.

$$
\begin{aligned}
& \text { (i) } \quad \mathcal{L}_{\left({ }^{b} a\right)}=\mathcal{L}_{a}, \forall a, b \in G ; \quad \text { (ii) } \quad \mathcal{L}_{\left(a^{b}\right)}=\mathcal{L}_{a}, \forall a, b \in G \text {; } \\
& \text { (iii) } \mathcal{R}_{\left({ }^{b} a\right)}=\mathcal{R}_{a}, \forall a, b \in G ; \quad \text { (iv) } \mathcal{R}_{\left(a^{b}\right)}=\mathcal{R}_{a}, \forall a, b \in G \text {. }
\end{aligned}
$$

Moreover, each of these four condition implies conditions lri and cc on $(G, r)$.

Proof. The implications (i) $\Longleftrightarrow$ (ii) $\Longrightarrow$ (condition lri) are verified by Lemma 7.1 for arbitrary symmetric sets. An argument analogous to the proof of Lemma 7.1 verifies (iii) $\Longleftrightarrow$ (iv) $\Longrightarrow$ (condition lri).

(i) $\Longrightarrow$ (iv). Suppose (i) holds, then (ii) is also in force. Let $a, b, c \in G$. We use successively condition lri, Lemma 7.5, and (ii) to obtain

$$
c^{a^{b}}={ }^{\left(a^{b}\right)^{-1}} c={ }^{\left(a^{-1}\right)^{b}} c={ }^{a^{-1}} c=c^{a},
$$

which implies (iv). One verifies (iv) $\Longrightarrow$ (i) analogously.

Proposition 7.13. Let $(G, r)$ be a symmetric group, and let $G=(G,+, \cdot)$ be the associated left brace. Suppose $(G, r)$ is a nontrivial solution. The following three conditions are equivalent.

(1) $(G, r)$ is a multipermutation solution of YBE of level 2.

(2) $G$ acts upon itself from the left as automorphisms, that is, MLaut, 7.14) holds.

(3) At least one of the four identities (7.20) (i), (ii), (iii), (iv) is in force.

Moreover, each of the above conditions implies the following.

(i) The symmetric set $(G, r)$ satisfies lri and the cyclic conditions $\boldsymbol{c c}$.

(ii) The brace $G=(G,+, \cdot)$ satisfies condition Raut.

Proof. (11) $\Longleftrightarrow$ (3). The equivalence (11) $\Longleftrightarrow$ (7.20) (ii) follows from Lemma 4.43. Hence, by Lemma 7.12, condition (11) is equivalent to each of the four conditions listed in (7.20). (2) $\Longleftrightarrow(3)$. We shall verify $(17.14) \Longleftrightarrow(7.20)$ (i). The symmetric group $G$ satisfies ML2, so ${ }^{a}(b c)=\left({ }^{a} b\right)\left(\left(a^{b}\right) c\right), \forall a, b, c \in G$. Therefore the following implications are in force:

$$
\begin{aligned}
(7.14) & \Longleftrightarrow\left[\left({ }^{a} b\right)\left({ }^{a} c\right)={ }^{a}(b c)=\left({ }^{a} b\right)\left({ }^{\left(a^{b}\right)} c\right), \forall a, b, c \in G\right] \\
& \Longleftrightarrow\left[{ }^{\left(a^{b}\right)} c={ }^{a} c, \forall a, b, c \in G\right] .
\end{aligned}
$$

This proves the equivalence (7.14) $\Longleftrightarrow(7.20)$ (i). Our argument above shows that conditions (2) and (3) are equivalent. It follows then that (1), (2) and (3) are equivalent, and by Lemma 7.12 each of them implies lri, hence, by Corollary 2.8 the cyclic conditions cc are also in force. By Theorem 7.10 (1) lri implies Raut.

Remark 7.14. Let $(G, r)$ be a symmetric group which acts upon itself as automorphisms, i.e. MLaut holds. Then the semidirect product $G \ltimes G$ is also a symmetric group, with a braiding operator canonically extending $r$.

This agrees with analogous statement of Rump about braces, see [Ru14, Proposition 6.2. 
7.4. The symmetric groups $\left(G, r_{G}\right)$ and $\left(\mathcal{G}, r_{\mathcal{G}}\right)$ of a nontrivial permutation solution. We consider the class of involutive permutation solutions of Lyubashenko (or shortly, the permutation solutions) : $(X, r)=(X, r, \sigma)$, where $X$ is a set, not necessarily finite, $|X| \geq 2, \sigma \in \operatorname{Sym}(X)$ is a permutation (bijective map), and $r$ is the map $r: X \times X \longrightarrow X \times X$ defined as $r(x, y):=\left(\sigma(y), \sigma^{-1}(x)\right), \forall x, y \in X$. $(X, r)$ satisfies lri, and is a nontrivial solution iff $\sigma \neq i d_{X}$. Recall that a symmetric set $(X, r)$ has $\mathrm{mpl} X=1$ iff $(X, r)$ is a permutation solution, see Remark 4.2. Moreover, $\operatorname{mpl} G(X, r)=\operatorname{mpl} X=1$ iff $\sigma=i d_{X}$, and $(X, r)$ is the trivial solution. The proof of the following proposition is given in our preprint [GI15, p. 40.

Proposition 7.15. Let $(X, r)=(X, r, \sigma)$ be the permutation solution defined by $\sigma \in \operatorname{Sym}(X), \sigma \neq i d_{X}$. Let $\left(G, r_{G}\right)$ and $\left(\mathcal{G}, r_{\mathcal{G}}\right)$ be the associated symmetric groups. Let $x \in X$. Then the following conditions hold.

(1) $\mathcal{G} \cong\langle\sigma\rangle$. The retraction $\operatorname{Ret}\left(G, r_{G}\right)=\left([G], r_{[G]}\right)$ is a trivial solution of order $|\sigma|$.

(2) More precisely, either (i) the permutation $\sigma$ has finite order $m$, then $\operatorname{Ret}\left(G, r_{G}\right)$ is the trivial solution on the set $[G]=\left\{[1],[x],\left[x^{2}\right],\left[x^{3}\right], \cdots\left[x^{m-1}\right]\right\}$; or (ii) the permutation $\sigma$ has infinite order, then $\operatorname{Ret}\left(G, r_{G}\right)$ is the trivial solution on the countably infinite set: $[G]=\left\{\cdots,\left[x^{-2}\right],\left[x^{-1}\right],[1],[x],\left[x^{2}\right], \cdots\right\}$.

(3) $G$ acts upon itself as automorphisms, that is conditions MLaut holds. Moreover, $G$ satisfies conditions lri and Raut.

(4) There is a strict inequality $1=\operatorname{mpl} X<\operatorname{mpl} G=2$.

Clearly if $(X, r)$ is a permutation solution of finite order, then the permutation $\sigma \in \operatorname{Sym}(X)$ has finite order $m$, so $\operatorname{Ret}\left(G, r_{G}\right)$ is a finite (trivial) symmetric set of order $m$. We give two concrete examples in which $X$ is a countably infinite set.

Example 7.16. Let $X=\left\{x_{i} \mid i \in \mathbb{Z}\right\}$. We consider the following two solutions.

(1) $\left(X, r, \sigma_{1}\right)=\left(X, r_{1}\right)$, where $\sigma_{1} \in \operatorname{Sym}(X)$ is the shift $\sigma_{1}\left(x_{i}\right)=x_{i+1}, i \in \mathbb{Z}$. Denote by $\left(G^{(1)}, r^{(1)}\right)$ the associated symmetric group. The permutation $\sigma_{1}$ has infinite order, $\operatorname{Ret}\left(G^{(1)}, r^{(1)}\right)$ is the trivial solution on the countably infinite set $\left[G^{(1)}\right]=\left\{\cdots,\left[x^{-2}\right],\left[x^{-1}\right],[1],[x],\left[x^{2}\right],\left[x^{3}\right], \cdots\right\}$, where $x \in X$ is an arbitrary fixed element of $X$.

(2) $\left(X, r, \sigma_{2}\right)=\left(X, r_{2}\right)$, where $\sigma_{2} \in \operatorname{Sym}(X)$ is the infinite product of transpositions $\sigma_{2}=\prod_{k \in \mathbb{Z}}\left(x_{2 k} x_{2 k+1}\right)$. In other words, $\sigma\left(x_{2 k}\right)=x_{2 k+1}, \sigma\left(x_{2 k+1}\right)=$ $x_{2 k}$, for all $k \in \mathbb{Z}$, so $\sigma^{2}=i d_{X}$. Denote by $\left(G^{(2)}, r^{(2)}\right)$ the associated symmetric group. Then $\operatorname{Ret}\left(G^{(2)}, r^{(2)}\right)$ is a trivial solution of order 2 , one has: $\left[G^{(2)}\right]=\{[1],[x]\}$, where $x \in X$ is an arbitrary fixed element of $X$.

In both cases there are equalities $\operatorname{mpl}\left(X, r_{i}\right)=1, \operatorname{mpl}\left(G^{(i)}, r^{(i)}\right)=2, i=1,2$.

\section{Square-Free solutions $(X, r)$ With CONDition lRi ON A DERIVED SYMMETRIC GROUP, OR ON A DERIVED PERMUTATION GROUP}

In this section $(X, r)$ denotes a square-free solution of arbitrary cardinality $|X| \geq 2$. We shall indicate explicitly the cases when $X$ is a finite set. As usual $\left(G, r_{G}\right)$ and $(G,+, \cdot)$ are the associated symmetric group and the corresponding left brace. Let $X_{i}, i \in I$, be the set of all $G$-orbits in $X$ (we consider the left action of $G$ upon $X$ ), $\left(X_{i}, r_{i}\right), i \in I$, denotes the induced solution on $X_{i}$. 
8.1. The symmetric group $G(X, r)$ satisfies lri if and only if $m p l X \leq 2$. The main result of the subsection is Theorem 8.2 We first study how condition lri on the associated symmetric group $\left(G, r_{G}\right)$ affects the properties of $(X, r)$.

Proposition 8.1. If $(X, r)$ is a nontrivial square-free solution, whose associated symmetric group $\left(G, r_{G}\right)$ satisfies condition lri, then the following conditions hold.

(1) For each $G$-orbit $X_{i}, i \in I$, the induced solution $\left(X_{i}, r_{i}\right)$, is a trivial, or one element solution.

(2) $\operatorname{mpl}(X, r)=\operatorname{mpl}\left(G, r_{G}\right)=2$.

Proof. (1). We shall verify the equalities

$$
\left.{ }^{b} x\right) x=x, \quad x^{\left({ }^{b} x\right)}=x, \quad \text { for all } b \in G, x \in X .
$$

By Theorem 7.10 (1) condition lri implies

$$
\left({ }^{\left({ }^{b} v\right)} u\right)\left({ }^{v} b\right)=\left({ }^{v} u\right)\left({ }^{\left(v^{u}\right)} b\right), \quad \forall b, u, v \in G .
$$

We set $u=x, v=x$ in this equality to obtain $\left.\left({ }^{b} x\right) x\right)\left({ }^{x} b\right)=\left({ }^{x} x\right)\left(\left({ }^{x}\right) b\right)=x\left({ }^{x} b\right)$ which, after cancelling ${ }^{x} b$ from both sides, implies the first equality in (8.1). We use lri to deduce the second equality in (8.1). Clearly, (8.1) implies $r(x, y)=\left({ }^{x} y, x^{y}\right)=$ $(y, x)$, for every $x, y \in X_{i}$, so $\left(X_{i}, r_{i}\right), i \in I$, is a trivial, or one element solution. (2). We shall prove $\operatorname{mpl} X=2$. By hypothesis $(X, r)$ is a nontrivial (square-free) solution. Let $a, x, y \in X$. Then ${ }^{(y a)} x,{ }^{a} y,{ }^{a} x,{ }^{\left(a^{x}\right)} y \in X$, so writing (8.2) in terms of $a, x, y$ we obtain the following equality of words of length two in $S=S(X, r)$ :

$$
\left({ }^{\left({ }^{y} a\right)} x\right)\left({ }^{a} y\right)=\left({ }^{a} x\right)\left({ }^{\left(a^{x}\right)} y\right) .
$$

Two cases are possible. Case i. The equality (8.3) holds in the free monoid $\langle X\rangle$. In this case $\left({ }^{(y a)} x,{ }^{a} y\right)=\left({ }^{a} x,{ }^{\left(a^{x}\right)} y\right)$ in $X \times X$, hence ${ }^{\left({ }^{y} a\right)} x={ }^{a} x$. Case ii. $\left.\left({ }^{y} a\right) x\right)\left({ }^{a} y\right) \neq\left({ }^{a} x\right)\left({ }^{\left(a^{x}\right)} y\right)$ in $\langle X\rangle$, so (8.3) is a non-trivial relation in $S$. Then

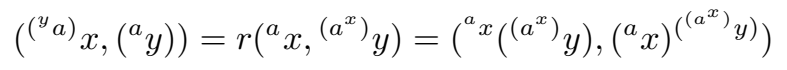

is an equality in $X \times X$. Comparing the first components we get

$$
\left.{ }^{(y} a\right) x={ }^{a} x\left({ }^{\left(a^{x}\right)} y\right),
$$

so $x$ and $y$ are in the same orbit $X_{i}$, for some $i$. Now (8.5) together with (8.3) and the fact that $\left(X_{i}, r_{i}\right), i \in I$, is a trivial solution imply

$$
\left.{ }^{a} x={ }^{y} a\right) x={ }^{a} x\left({ }^{\left(a^{x}\right)} y\right)={ }^{\left(a^{x}\right)} y={ }^{a} y .
$$

Thus ${ }^{a} x={ }^{a} y$, which by the non-degeneracy implies $x=y$, and therefore (ii) is impossible. We have shown that ${ }^{(y a)} x={ }^{a} x$, for all $a, x, y \in X$, or equivalently, $\mathcal{L}_{\left({ }^{y} a\right)}=\mathcal{L}_{a}, \quad \forall a, y \in X$. This implies that $\operatorname{mpl} X=2$. By hypothesis $(X, r)$ is a square-free solution, so Theorem 5.15 implies $\operatorname{mpl} G=\operatorname{mpl} X=2$.

Theorem 8.2. Let $(X, r)$ be a nontrivial square-free solution of arbitrary cardinality.

(1) The following conditions are equivalent

(a) $(X, r)$ is a multipermutation solution of level 2 .

(b) $\left(G, r_{G}\right)$ is a multipermutation solution of level 2 .

(c) $G$ acts upon itself as automorphisms that is $\mathcal{L}_{\left({ }^{b} a\right)}=\mathcal{L}_{a}, \quad \forall a, b \in G$.

(d) $\left(G, r_{G}\right)$ satisfies condition lri. 
(e) The left brace $(G,+, \cdot)$ satisfies condition Raut.

(2) More generally, $\operatorname{mpl}(X, r)=m \geq 2$ iff the derived group $\left(G_{m-2}, r_{G_{m-2}}\right)$ is nonabelian and satisfies lri.

Proof. (1). The implication (1a) $\Longleftrightarrow(1 \mathrm{~b})$ follows from Theorem 5.15 part (3). Note that each of the solutions $(X, r)$ and $\left(G, r_{G}\right)$ satisfies condition $\left(^{*}\right)$, see Definition 4.3. Then Lemma 4.4 part (3) verifies the implications $(1 b) \Longleftrightarrow(1 c) \Longrightarrow(1 d)$. Proposition 8.1 verifies (1d) $\Longrightarrow$ (1ad). Since every square-free solution $(X, r)$ satisfies lri, Corollary 7.11 gives the implication (1d) $\Longleftrightarrow$ (1e). (2). By definition $\left(G_{m-2}, r_{G_{m-2}}\right)=G\left(\operatorname{Ret}^{m-2}(X, r)\right)$, moreover there are equivalences:

$$
[\operatorname{mpl}(X, r)=m \geq 2] \Longleftrightarrow\left[\operatorname{mpl} \operatorname{Ret}^{m-2}(X, r)=2\right] \Longleftrightarrow\left[\operatorname{mpl}\left(G_{m-2}, r_{G_{m-2}}\right)=2\right] \text {. }
$$

Part (1) implies mpl $\operatorname{Ret}^{m-2}(X, r)=2$ iff $\left(G_{m-2}, r_{G_{m-2}}\right)$ satisfies lri and is nonabelian, which verifies part (2).

8.2. A finite square-free symmetric set $(X, r)$ whose permutation group $\mathcal{G}(X, r)$ satisfies lri is a multipermutation solution. The conventions given in the beginning of this section are in force. So $(X, r)$ denotes a nontrivial squarefree solution of arbitrary cardinality, $|X| \geq 2,\left(G, r_{G}\right),\left(\mathcal{G}, r_{\mathcal{G}}\right)$ are the associated symmetric groups, $S=S(X, r)$ is the associated monoid, $\left(G_{j}, r_{G_{j}}\right)$, and $\left(\mathcal{G}_{j}, r_{\mathcal{G}}\right)$, $j \geq 0$ are the corresponding derived groups. In particular, $G_{1}=G\left([X], r_{[X]}\right)$ is the symmetric group of the retraction $\operatorname{Ret}(X, r)=\left([X], r_{[X]}\right)$. We denote by $X_{i}, i \in I$, the set of all $G$-orbits in $X$ (possibly infinite) and by $\left(X_{i}, r_{i}\right), i \in I$, the induced solutions. Clearly, a subset $Y \subset X$ is a $G$-orbit iff it is a $\mathcal{G}$-orbit. The main result of the subsection is Theorem 8.5

Consider the group epimorphism $\mathcal{L}: G \longrightarrow \mathcal{G}$, extending the assignment $x \mapsto$ $\mathcal{L}_{x}, x \in X$. For $a \in G$ we shall often write $\bar{a}:=\mathcal{L}_{a} \in \mathcal{G}$, so every element $u \in \mathcal{G}$ can be written as $u=\bar{a}$, for some $a \in G$. If $a, b \in G$ we write $a \sim b$ to express that $\mathcal{L}_{a}=\mathcal{L}_{b}$ on $X$ (by Remark 5.1 this implies equality of the left actions $\mathcal{L}_{a}=\mathcal{L}_{b}$ of $G$ upon itself). We shall involve again computation with long actions, or, as we call these, "towers of actions".

Lemma 8.3. Notation as above. Let $(X, r)$ be a nontrivial solution, $|X| \geq 2$, such that: (i) for each $i \in I$, the $G$-orbit $\left(X_{i}, r_{i}\right)$ is a trivial solution, or one element solution; and (ii) the symmetric group $\left(\mathcal{G}, r_{\mathcal{G}}\right)$ satisfies condition lri. Then the equality:

$$
\left(\left(\cdots\left(\left(b \triangleright y_{n}\right) \triangleright y_{n-1}\right) \triangleright \cdots \triangleright y_{2}\right) \triangleright y_{1}\right) \triangleright a=\left(\left(\cdots\left(y_{n} \triangleright y_{n-1}\right) \triangleright \cdots \triangleright y_{2}\right) \triangleright y_{1}\right) \triangleright a
$$

holds for any integer $n \geq 1$, any choice of $y_{1}, \cdots, y_{n} \in X$, and any pair $a, b$, such that $a \in X_{i}, b \in X_{i} \bigcup X_{i}^{-1}, i \in I$.

Proof. By hypothesis $\left(\mathcal{G}, r_{\mathcal{G}}\right)$ satisfies condition lri, hence by Theorem 7.10 , the identity (7.16) hods in $\mathcal{G}$. We write it using the alternative notation, " $\alpha \triangleright x={ }^{\alpha} x "$ :

$$
((v \triangleright u) \triangleright b)(u \triangleright v)=(u \triangleright b) \cdot\left(\left(u^{b}\right) \triangleright v\right), \quad \forall u, v, b \in \mathcal{G} .
$$

Equivalently, one has

$$
\overline{((v \triangleright u) \triangleright b)(u \triangleright v)}=\overline{(u \triangleright b) \cdot\left(\left(u^{b}\right) \triangleright v\right)}, \quad \forall u, v, b \in G .
$$

We shall use induction on $n$ to prove that (8.6) holds for any $n$, any choice of $y_{1}, \cdots, y_{n} \in X$, and any $a$ and $b$ such that $a \in X_{i}, b \in X_{i} \bigcup X_{i}^{-1}, i \in I$. The 
base for the induction follows straightforwardly from Proposition 7.2. (7.2). Due to condition lri on $(X, r)$, the equalities (7.2) are satisfied for all $y \in X$, and all $a, b$, such that $a \in X_{i}, b \in X_{i} \bigcup X_{i}^{-1}, i \in I$. Assume (8.6) is in force for all $n, 1 \leq n \leq k$. Chose $y_{1}, \cdots, y_{k}, y_{k+1} \in X, a$ and $b$ as above. We write (8.7) in terms of $y_{k}, y_{k+1}, b$ to obtain

$$
\left(\overline{\left(y_{k} \triangleright y_{k+1}\right) \triangleright b}\right) \cdot\left(\overline{y_{k+1} \triangleright y_{k}}\right)=\left(\overline{y_{k+1} \triangleright b}\right) \cdot\left(\overline{\left(y_{k+1}^{b}\right) \triangleright y_{k}}\right) .
$$

This implies an equivalence of elements of $G$ with respect to their left action upon $X$ :

$$
\left(\left(y_{k} \triangleright y_{k+1}\right) \triangleright b\right) \cdot\left(y_{k+1} \triangleright y_{k}\right) \sim\left(y_{k+1} \triangleright b\right) \cdot\left(\left(y_{k+1}^{b}\right) \triangleright y_{k}\right) .
$$

Now we set

$$
\begin{array}{ll}
b^{\prime}=\left(y_{k} \triangleright y_{k+1}\right) \triangleright b, & y_{k}{ }^{\prime}=y_{k+1} \triangleright y_{k}, \\
b^{\prime \prime}=y_{k+1} \triangleright b, & y_{k}{ }^{\prime \prime}=\left(y_{k+1}^{b}\right) \triangleright y_{k} .
\end{array}
$$

Substitute these in (8.9) and obtain $b^{\prime} \cdot y_{k}{ }^{\prime} \sim b^{\prime \prime} \cdot y_{k}{ }^{\prime \prime}$. Recall that $G$ acts on $X^{*}$, see Section 7.1. By Proposition 7.6 one has $\left({ }^{c} b\right)^{-1}={ }^{c}\left(b^{-1}\right), \forall c \in G, b \in X^{*}$, hence $b^{-1} \in X_{i}$ implies $\left({ }^{c} b\right)^{-1} \in X_{i}, \forall c \in G$. Therefore each of the pairs $a, b^{\prime}$ and $a, b^{\prime \prime}$ satisfies the inductive assumption.

Without loss of generality we may assume that $k \geq 2$. (In the case when $k=1$, replace $y_{k-1}$ by $a$ in the argument). Then acting on the left upon $y_{k-1}$ we yield $\left(b^{\prime} \cdot y_{k}{ }^{\prime}\right) \triangleright y_{k-1}=\left(b^{\prime \prime} \cdot y_{k}^{\prime \prime}\right) \triangleright y_{k-1}$, which by condition ML1 gives

$$
\omega=b^{\prime} \triangleright\left(y_{k}^{\prime} \triangleright y_{k-1}\right)=b^{\prime \prime} \triangleright\left(y_{k}^{\prime \prime} \triangleright y_{k-1}\right) .
$$

Using these two presentations of the element $\omega$ we have the following equalities of "towers of actions" denoted by $\tau$ and $\tau_{1}$

$$
\begin{aligned}
\tau & =\left(\cdots\left(\left(b^{\prime} \triangleright\left(y_{k}^{\prime} \triangleright y_{k-1}\right)\right) \triangleright y_{k-2}\right) \triangleright \cdots \triangleright y_{1}\right) \triangleright a \\
& =\left(\left(\cdots\left(b^{\prime \prime} \triangleright\left(y_{k}^{\prime \prime} \triangleright y_{k-1}\right)\right) \triangleright y_{k-2}\right) \triangleright \cdots \triangleright y_{1}\right) \triangleright a=\tau_{1} .
\end{aligned}
$$

Next we apply the inductive assumption to each of the elements $\tau$ and $\tau_{1}$ occurring in (8.11). For $\tau$ we have

$$
\begin{aligned}
\tau & =\left(\cdots\left(\left(b^{\prime} \triangleright\left(y_{k}^{\prime} \triangleright y_{k-1}\right)\right) \triangleright y_{k-2}\right) \triangleright \cdots \triangleright y_{1}\right) \triangleright a & \\
& =\left(\cdots\left(\left(y_{k}^{\prime} \triangleright y_{k-1}\right) \triangleright y_{k-2}\right) \triangleright \cdots \triangleright y_{1}\right) \triangleright a & \text { by IH } \\
& =\left(\cdots\left(\left(y_{k+1} \triangleright y_{k}\right) \triangleright y_{k-1}\right) \triangleright \cdots \triangleright y_{1}\right) \triangleright a & \text { by }(\underline{8.10}),
\end{aligned}
$$

where IH is the inductive hypothesis. Similarly, for $\tau_{1}$ we have

$$
\begin{aligned}
\tau_{1} & =\left(\left(\cdots\left(b^{\prime \prime} \triangleright\left(y_{k}{ }^{\prime \prime} \triangleright y_{k-1}\right)\right) \triangleright y_{k-2}\right) \cdots \triangleright y_{1}\right) \triangleright a & & \\
& =\left(\cdots\left(\left(y_{k}{ }^{\prime \prime} \triangleright y_{k-1}\right) \triangleright y_{k-2}\right) \cdots \triangleright y_{1}\right) \triangleright a & & \text { by IH } \\
& =\left(\cdots\left(\left(\left(\left(y_{k+1}^{b}\right) \triangleright y_{k}\right) \triangleright y_{k-1}\right) \triangleright y_{k-2}\right) \triangleright \cdots \triangleright y_{1}\right) \triangleright a & & \text { by (8.10) } \\
& =\left(\cdots\left(\left(\left(\left(b^{-1} \triangleright y_{k+1}\right) \triangleright y_{k}\right) \triangleright y_{k-1}\right) \triangleright y_{k-2}\right) \triangleright \cdots \triangleright y_{1}\right) \triangleright a & & \text { by lri } \\
& =\left(\cdots\left(\left(\left(\left(c \triangleright y_{k+1}\right) \triangleright y_{k}\right) \triangleright y_{k-1}\right) \triangleright y_{k-2}\right) \triangleright \cdots \triangleright y_{1}\right) \triangleright a, & & \text { where } c=b^{-1} .
\end{aligned}
$$

The presentations of $\tau_{1}$ and $\tau$ given in (8.13) and (8.12), together with $\tau_{1}=\tau$ imply that the equality

$$
\left(\cdots\left(\left(\left(c \triangleright y_{k+1}\right) \triangleright y_{k}\right) \triangleright y_{k-1}\right) \triangleright \cdots \triangleright y_{1}\right) \triangleright a=\left(\cdots\left(\left(y_{k+1} \triangleright y_{k}\right) \triangleright y_{k-1}\right) \triangleright \cdots \triangleright y_{1}\right) \triangleright a
$$

holds, whenever $a \in X_{i}$ and $c \in X_{i} \bigcup X_{i}^{-1}, i \in I$. 
Proposition 8.4. Let $(X, r)$ be a nontrivial solution, whose symmetric group $\left(\mathcal{G}, r_{\mathcal{G}}\right)$ satisfies condition lri. Suppose $X$ has a finite number of $\mathcal{G}$-orbits $X_{i}, 1 \leq$ $i \leq m$, and that each $\left(X_{i}, r_{i}\right), 1 \leq i \leq m$, is a trivial solution, or one element solution. Then $(X, r)$ is a square-free multipermutation solution, with $|X| \geq 3$, $2 \leq \operatorname{mpl}(X, r) \leq m$ and presents as a strong twisted union $X=X_{1} \curvearrowleft X_{2} \cdots \bullet X_{m}$ of solutions with $0 \leq \operatorname{mpl}\left(X_{i}\right) \leq 1$.

Proof. It is clear that $(X, r)$ is a square-free solution, since each orbit is a trivial solution and therefore a square-free symmetric set. But a square-free solution of order $|X|=2$ is trivial, hence the hypothesis of the proposition implies $|X| \geq 3$. It follows from Proposition 7.2 that $X$ is a strong twisted union $X=X_{1} \curvearrowleft X_{2} \cdots \curvearrowleft X_{m}$ of solutions with $0 \leq \operatorname{mpl}\left(X_{i}\right) \leq 1$. We shall prove that $(X, r)$ is a multipermutation solution, with $2 \leq \operatorname{mpl}(X, r) \leq m$. The square-free solution $(X, r)$ satisfies the hypothesis of Proposition 4.7. (2), therefore it will be enough to show that

$$
\begin{aligned}
\omega= & \left(\cdots\left(\left(y_{m+1} \triangleright y_{m}\right) \triangleright y_{m-1}\right) \triangleright \cdots \triangleright y_{2}\right) \triangleright y_{1} \\
= & \left(\cdots\left(y_{m} \triangleright y_{m-1}\right) \triangleright \cdots \triangleright y_{2}\right) \triangleright y_{1}=\omega^{\prime}, \\
& \text { for all } y_{1}, \cdots, y_{m+1} \in X .
\end{aligned}
$$

Let $y_{1}, \cdots, y_{m+1} \in X$. There are exactly $m$ orbits, hence there will be some integers $\lambda, \mu, 1 \leq \lambda<\lambda+\mu \leq m+1$, such that $y_{\lambda}, y_{\lambda+\mu}$ are in the same orbit, say $X_{i}$. Two cases are possible. Case 1. $\mu=1$. In this case $\lambda+\mu=\lambda+1$, so $\left(\cdots\left(y_{m+1} \triangleright y_{m}\right) \triangleright \cdots\right) \triangleright y_{\lambda+1}=u \in X_{i}$, and since $X_{i}$ is a trivial solution, one has $\left(\cdots\left(y_{m+1} \triangleright y_{m}\right) \triangleright \cdots \triangleright y_{\lambda+1}\right) \triangleright y_{\lambda}={ }^{u} y_{\lambda}=y_{\lambda}$. Thus

$$
\omega=\left(\cdots\left(\left(y_{m+1} \triangleright y_{m}\right) \triangleright y_{m-1}\right) \triangleright \cdots \triangleright y_{2}\right) \triangleright y_{1}=\left(\cdots\left(y_{\lambda} \triangleright y_{\lambda-1}\right) \triangleright \cdots \triangleright y_{2}\right) \triangleright y_{1} .
$$

Similarly, $\omega^{\prime}=\left(\cdots\left(y_{m} \triangleright y_{m-1}\right) \triangleright \cdots \triangleright y_{2}\right) \triangleright y_{1}=\left(\cdots\left(y_{\lambda} \triangleright y_{\lambda-1}\right) \triangleright \cdots \triangleright y_{2}\right) \triangleright y_{1}$. This gives the desired equality $\omega=\omega^{\prime}$, which proves (8.14). Case 2. $\mu>1$. We shall assume $\lambda+\mu<m+1$ (The proof in the case $\lambda+\mu=m+1$ is analogous). We set

$$
\begin{aligned}
& z=y_{\lambda}, \quad u=y_{\lambda+\mu}, \quad u^{\prime}=\left(\cdots\left(y_{m+1} \triangleright y_{m}\right) \triangleright \cdots\right) \triangleright y_{\lambda+\mu}, \\
& \omega_{\lambda}=\left(\left(\cdots\left(u^{\prime} \triangleright y_{\lambda+\mu-1}\right) \triangleright y_{\lambda+\mu-2}\right) \cdots \triangleright y_{\lambda+1}\right) \triangleright z .
\end{aligned}
$$

In this notation $\omega$ presents as

$$
\omega=\left(\cdots\left(\omega_{\lambda} \triangleright y_{\lambda-1}\right) \triangleright \cdots\right) \triangleright y_{1} .
$$

By assumption $u=y_{\lambda+\mu} \in X_{i}$, hence $u^{\prime}$ is also in $X_{i}$. The tower $\omega_{\lambda}$ contains the elements $u^{\prime}$ and $z$ which belong to the same orbit $X_{i}$, therefore by Lemma 8.3 we can "cut" $u^{\prime}$ to yield

$$
\begin{aligned}
\omega_{\lambda} & =\left(\left(\cdots\left(u^{\prime} \triangleright y_{\lambda+\mu-1}\right) \triangleright y_{\lambda+\mu-2}\right) \cdots \triangleright y_{\lambda+1}\right) \triangleright z=\left(\cdots\left(y_{\lambda+\mu-1} \triangleright y_{\lambda+\mu-2}\right) \cdots \triangleright y_{\lambda+1}\right) \triangleright z \\
& =\left(\cdots\left(y_{\lambda+\mu-1} \triangleright y_{\lambda+\mu-2}\right) \cdots \triangleright y_{\lambda+1}\right) \triangleright y_{\lambda} .
\end{aligned}
$$

We use this equality and (8.16) to yield a truncation of $\omega$ :

$$
\begin{aligned}
\omega & =\left(\cdots\left(\left(y_{m+1} \triangleright y_{m}\right) \triangleright y_{m-1}\right) \triangleright \cdots \triangleright y_{2}\right) \triangleright y_{1}=\left(\cdots\left(\omega_{\lambda} \triangleright y_{\lambda-1}\right) \triangleright \cdots\right) \triangleright y_{1} \\
& =\left(\left(\cdots\left(\left(\cdots\left(y_{\lambda+\mu-1} \triangleright y_{\lambda+\mu-2}\right) \cdots \triangleright y_{\lambda+1}\right) \triangleright y_{\lambda}\right) \triangleright \cdots\right) \triangleright y_{2}\right) \triangleright y_{1} .
\end{aligned}
$$

Analogous argument verifies that the tower $\omega^{\prime}$ of length $m$ can also be truncated:

$$
\begin{aligned}
\omega^{\prime} & =\left(\cdots\left(y_{m} \triangleright y_{m-1}\right) \triangleright \cdots \triangleright y_{2}\right) \triangleright y_{1} \\
& =\left(\left(\cdots\left(\left(\cdots\left(y_{\lambda+\mu-1} \triangleright y_{\lambda+\mu-2}\right) \cdots \triangleright y_{\lambda+1}\right) \triangleright y_{\lambda}\right) \triangleright \cdots\right) \triangleright y_{2}\right) \triangleright y_{1}=\omega .
\end{aligned}
$$

Therefore (8.14) is in force, and Proposition 4.7 implies mpl $G \leq m$. 
Theorem 8.5. Let $(X, r)$ be a square-free solution, $|X| \geq 2$, with associated permutation group $\mathcal{G}=\mathcal{G}(X, r)$. Suppose that the symmetric group $\left(\mathcal{G}, r_{\mathcal{G}}\right)$ satisfies lri. Then the following is in force.

(1) At least one of the following two conditions holds: (a) The solution $(X, r)$ is retractable; (b) Each induced solution $\left(X_{i}, r_{i}\right), i \in I$, is a trivial solution, or one element solution and $X$ is a strong twisted union $X=\natural_{i \in I} X_{i}$.

(2) If $X$ is a finite set, then $(X, r)$ is a multipermutation solution.

Proof. (1). By hypothesis $\left(\mathcal{G}, r_{\mathcal{G}}\right)$ satisfies condition lri, hence the equality (8.7) holds for all $u, v, b \in G$. We choose $x \in X, v \in G$, set $b=x, u=x$, and replace these in (8.7). Then the following equalities hold for all $x \in X, v \in G$ :

$$
(\overline{(v \triangleright x) \triangleright x}) \cdot\left(\overline{x \triangleright v)}=\left(\overline{(v \triangleright x) \triangleright x)(x \triangleright v)}=\overline{(x \triangleright x) \cdot\left(\left(x^{x}\right) \triangleright v\right)}=\overline{x \cdot(x \triangleright v)}=\bar{x} \cdot \overline{(x \triangleright v)} .\right.\right.
$$

Now cancel $\overline{x \triangleright v}$ from the first and from the last monomial to obtain $\overline{((v \triangleright x) \triangleright x)}=\bar{x}$, for all $x \in X$ and all $v \in G$. This implies the equality of actions

$$
\mathcal{L}_{((v \triangleright x) \triangleright x)}=\mathcal{L}_{x}, \quad \forall x \in X, v \in G .
$$

Two cases are possible. Case (i). There exist $x \in X$ and $v \in G$ such that

$$
(v \triangleright x) \triangleright x \neq x \text {, but } \mathcal{L}_{((v \triangleright x) \triangleright x)}=\mathcal{L}_{x} .
$$

This implies that $(X, r)$ is retractable. Case (ii). $(v \triangleright x) \triangleright x=x$ for all $x \in X$, and all $v \in G$. This is equivalent to

$$
{ }^{y} x=x, \quad \forall x, y \in X_{i}, i \in I .
$$

It follows that the induced solution $\left(X_{i}, r_{i}\right), i \in I$, is a trivial solution, or one element solution. The hypothesis of Proposition 8.4 is satisfied and therefore $X$ is a strong twisted union $X=\natural_{i \in I} X_{i}$, in the sense of GIC12. Part (11) has been proved. (2). Assume that $(X, r)$ is a finite square-free solution. Then it has a finite set of $\mathcal{G}$-orbits, say $X_{1}, \cdots X_{t}$. Now consider each of the two cases (i) and (ii) occurring in the argument of part (1). In the case when (ii) holds, each induced solution $\left(X_{i}, r_{i}\right), 1 \leq i \leq t$, is a trivial solution or one element solution. So Proposition 8.4 implies that $(X, r)$ is a multipermutation solution with $\operatorname{mpl} X \leq t$. Suppose case (i) is in force, then $(X, r)$ is retractable. Its retraction $\operatorname{Ret}(X, r)=\left([X], r_{[X]}\right)$ is a square-free symmetric set of order $<|X|$. The corresponding symmetric group $\mathcal{G}_{1}=$ $\mathcal{G}\left([X], r_{[X]}\right)$ is a (braiding-preserving) homomorphic image of $\left(\mathcal{G}, r_{\mathcal{G}}\right)$, so $\left(\mathcal{G}_{1}, r_{\mathcal{G}_{1}}\right)$ inherits condition lri from $\mathcal{G}$. It follows by induction on the order $|X|$ of $X$ that $(X, r)$ is a multipermutation solution with $\mathrm{mpl} X \leq|X|-1$.

Suppose $(X, r)$ a (nontrivial) square-free solution, $\left(G, r_{G}\right),\left(\mathcal{G}, r_{\mathcal{G}}\right)$ in usual notation. We have shown that the conditions "mpl $X=2$ " and " $\left(G, r_{G}\right)$ satisfies lri" are equivalent (Theorem 8.2). Moreover, condition lri on $\left(\mathcal{G}, r_{\mathcal{G}}\right)$ is sufficient for "mpl $X<\infty$ " but it does not give information about the precise value of $\mathrm{mpl} X$. We shall show that " $\left(\mathcal{G}, r_{\mathcal{G}}\right)$ satisfies lri" is a necessary condition for " $\mathrm{mpl} X=3$ ".

Lemma 8.6. Assumption and notation as above. Suppose $(X, r)$ is a square-free solution with $\mathrm{mpl} X=3$. Then $\left(\mathcal{G}, r_{\mathcal{G}}\right)$ satisfies lri and therefore Raut. 
Proof. It follows from Theorem 8.2 that $\mathrm{mpl} X=3$ if and only if the derived symmetric group $\left(G_{1}, r_{G_{1}}\right)$ satisfies condition lri and is a nontrivial solution. The canonical map $f:\left(G_{1}, r_{G_{1}}\right) \longrightarrow\left(\mathcal{G}, r_{\mathcal{G}}\right),[x] \mapsto \mathcal{L}_{x}$, see Lemma [5.4, is an epimorphism of symmetric groups, so $\left(\mathcal{G}, r_{\mathcal{G}}\right)$ also satisfies conditions lri and Raut.

Corollary 8.7. Suppose $(X, r)$ is a finite nontrivial square-free solution. The following conditions are equivalent.

(1) $2 \leq \operatorname{mpl} X=m<\infty$.

(2) There exists an integer $j \geq 0$, such that the derived permutation group $\left(\mathcal{G}_{j}, r_{\mathcal{G}_{j}}\right)$ satisfies lri, and $\mathcal{G}_{j} \neq\{1\}$.

Proof. If $\operatorname{mpl} X=2$, then $\operatorname{mpl}\left(\mathcal{G}, r_{\mathcal{G}}\right)=1$, so $\left(\mathcal{G}, r_{\mathcal{G}}\right)$ satisfies lri. Assume now $m=\operatorname{mpl} X \geq 3$. Then mpl $\operatorname{Ret}^{m-3}(X, r)=3$, and by Lemma 8.6 the derived permutation group $\mathcal{G}_{m-3}$ satisfies lri. Moreover, mpl $\mathcal{G}_{m-3}=\operatorname{mpl}_{\operatorname{Ret}^{m-3}}(X, r)-$ $1=2$, so $\mathcal{G}_{m-3} \neq\{1\}$. Conversely, if $\left(\mathcal{G}_{j}, r_{\mathcal{G}_{j}}\right)$ satisfies lri for some $j \geq 0$ and $\mathcal{G}_{j} \neq\{1\}$ then the corresponding retraction $\operatorname{Ret}^{j}(X, r)$ has finite multipermutation level, $2 \leq \operatorname{mpl} \operatorname{Ret}^{j}(X, r)=s$, hence $2 \leq \operatorname{mpl}(X, r)=j+s<\infty$.

\section{Epilogue}

Suppose $(X, r)$ is an arbitrary non-degenerate symmetric set, $|X| \geq 2$, no additional restrictions are imposed on $(X, r)$, unless specified explicitly. Let $\left(G, r_{G}\right),\left(\mathcal{G}, r_{\mathcal{G}}\right)$ be the associated symmetric groups, and let $\left(G_{j}, r_{G_{j}}\right)=G\left(\operatorname{Ret}^{j}(X, r)\right), j \geq 0, \mathcal{G}_{j}=$ $\mathcal{G}\left(\operatorname{Ret}^{j}(X, r)\right), j \geq 0$, be the derived symmetric groups, and the derived permutation groups related to $(X, r) . m \geq 1$ denotes an integer. We have verified the following.

(1) $[(G,+, \cdot)$ is a two sided brace $] \Longleftrightarrow[(X, r)$ is a trivial solution].

(2) $[\mathrm{mpl} G<\infty] \Longleftrightarrow[\operatorname{mpl} X<\infty] \Longleftrightarrow[\operatorname{mpl} \mathcal{G}<\infty]$. In this case

$$
0 \leq \operatorname{mpl} \mathcal{G}=m-1 \leq \operatorname{mpl} X \leq \operatorname{mpl} G=m<\infty .
$$

(3) If $(X, r)$ is square-free, then the following are equivalent: (i) $\operatorname{mpl} X=m$; (ii) $\mathrm{mpl} G=m$; (iii) $\left(G_{m-2}, r_{G_{m-2}}\right)$ satisfies lri and is not abelian; (iv) $\left(G_{m-2},+, \cdot\right)$ satisfies Raut and is not abelian; (v) the derived chain of ideals for $G$ has the shape $\{1\}=K_{0} \varsubsetneqq K_{1} \varsubsetneqq K_{2} \varsubsetneqq \cdots \varsubsetneqq K_{m-1} \varsubsetneqq K_{m}=G$; (vi) The brace $G$ is right nilpotent of nilpotency class $m+1$, i.e. $G^{(m+1)}=0$, and $G^{(m)} \neq 0$.

(4) If $X$ is finite then

$[2 \leq \operatorname{mpl} X<\infty] \Longleftrightarrow\left[\exists j \geq 0\right.$, such that $\left(\mathcal{G}_{j},+, \cdot\right)$ is a two-sided brace, $\left.\mathcal{G}_{j} \neq\{1\}\right]$.

(5) If $(X, r)$ is finite and square-free then

$$
[2 \leq \operatorname{mpl} X<\infty] \Longleftrightarrow\left[\exists j \geq 0 \text {, s.t. }\left(\mathcal{G}_{j}, r_{\mathcal{G}_{j}}\right) \text { satisfies lri, } \mathcal{G}_{j} \neq\{1\}\right] .
$$

Acknowledgements. This work was completed in 2014-2016 while I was visiting IHÉS, Max-Planck Institute for Mathematics, Bonn, and ICTP, Trieste. I thank Shahn Majid for our inspiring collaboration through the years and for introducing me to the fascinating theory of matched pairs of groups. It is my pleasant duty to thank Maxim Kontsevich and Don Zagier for several stimulating discussions. I give my cordial thanks to Agata Smoktunowicz and Ferran Cedó for our fruitful collaboration. 


\section{REFERENCES}

[AGV] Angiono, I, Galindo, C, Vendramin, L, Hopf braces and Yang-Baxter operators, arXiv preprint arXiv:1604.02098 [math.QA], 2016.

[BC] Bachiller D., Cedó F., A family of solutions of the Yang-Baxter equation J. Algebra, 412 (2014), 218-229.

[BCJ] Bachiller D., Cedó F., Jespers, E., Solutions of the Yang-Baxter equation associated with a left brace, J. Algebra, 463 (2016), 80-102.

[BCJO] Bachiller D., Cedó F., Jespers, E., Okniński, J. A family of irretractable square-free solutions of the Yang-Baxter equation arXiv:1511.07769] [math.GR] Oct. 2016.

[CES] Carter, J.S., Elhamdadi, M., Saito, M., Homology theory for the set-theoretic Yang-Baxter equation and knot invariants from generalizations of quandles, Fund. Math. 184 (2004), $31-54$.

[CGIS16] Cedó, F., Gateva-Ivanova, T, Smoktunowicz, A, On the Yang-Baxter equation and left nilpotent left braces, Journal of Pure and Applied Algebra, 221 (2017), 751-756. doi:10.1016/j.jpaa.2016.07.014

[CJO06] Cedó, F., Jespers, E. , Okniński J., The Gelfand-Kirillov dimension of quadratic algebras satisfying the cyclic condition, Proc. Amer. Math. Soc. 134 (2006), 653-663.

[CJO10] Cedó, F., Jespers, E., Okniński J., Retractability of set theoretic solutions of the Yang Baxter equation, Adv. in Math. 224 (2010), 2472-2484.

[CJO14] Cedó, F., Jespers, E., Okniński J., Braces and the Yang-Baxter equation, Commun. Math. Phys. 327, (2014) 101-116.

[De15] Dehornoy, P., Set-theoretic solutions of the Yang-Baxter equation, RC-calculus, and Garside germs, Adv. in Math. 282 (2015), 93-127.

[Dri] Drinfeld, V., On some unsolved problems in quantum group theory, Lecture Notes in Mathematics 1510 (1992), 1-8.

[ESS] Etingof, P., Schedler, T. and Soloviev, A., Set-theoretical solutions to the quantum YangBaxter equation, Duke Math. J. 100 (1999), 169-209.

[GI94] Gateva-Ivanova, T., Noetherian properties of skew polynomial rings with binomial relations, Trans. Amer. Math. Soc. 343 (1994), 203-219.

[GI96] Gateva-Ivanova, T., Skew polynomial rings with binomial relations, J. Algebra 185 (1996), $710-753$

[GIJO] Gateva-Ivanova, T., E. Jespers, and J. Okninski, Quadratic algebras of skew type and the underlying semigroups, J. Algebra 270 (2003), 635-659.

[GI04s] Gateva-Ivanova, T., Quantum binomial algebras, Artin-Schelter regular rings, and solutions of the Yang-Baxter equations, Serdica Math. J. 30 (2004), 431-470.

[GI04] Gateva-Ivanova, T., A combinatorial approach to the set-theoretic solutions of the YangBaxter equation, J.Math.Phys. 45 (2004), 3828-3858.

[GI11] T. Gateva-Ivanova, Garside structures on monoids with quadratic square-free relations, Algebr Represent Theor 14 (2011), 779-802.

[GI12] Gateva-Ivanova, T., Quadratic algebras, Yang-Baxter equation, and Artin- Schelter regularity, Adv. in Math. 230 (2012), 2152-2175.

[GI15] Tatiana Gateva-Ivanova, Set-theoretic solutions of the Yang-Baxter equation, Braces, and Symmetric groups, arXiv:1507.02602 [math.QA] v2. Mon, 31 Aug 2015, 1-47.

[GIB] Gateva-Ivanova, T. and Van den Bergh, M., Semigroups of I-type, J. Algebra 206 (1998), 97-112.

[GIC12] Gateva-Ivanova, T. and Cameron, P.J., Multipermutation solutions of the Yang-Baxter equation, Comm. Math. Phys. 309 (2012), 583-621.

[GIM07] Gateva-Ivanova, T. and Majid, S., Set Theoretic Solutions of the Yang-Baxter Equations, Graphs and Computations, J. Symb. Comp. 42 (2007), 1079-1112.

[GIM08] Gateva-Ivanova, T. and Majid, S., Matched pairs approach to set theoretic solutions of the Yang-Baxter equation, J. Algebra 319 (2008), 1462-1529.

[GIM11] T. Gateva-Ivanova, S. MajID, Quantum spaces associated to multipermutation solutions of level two, Algebr. Represent. Theor. 14 (2011) 341-376

[JO04] Jespers, E. , Okniński J., Quadratic algebras of skew type satisfying the cyclic condition, Int. J. Algebra and Computation 14 (2004), 479-498.

[KP66] Kac, G.I., Paljutkin, V.G., Finite ring groups, Trans. Amer. Math. Soc. 15 (1966) 251294. 
[Lam] Lam, T. Y. (2001), A first course in noncommutative rings, Graduate Texts in Mathematics 131 (2 ed.), New York: Springer-Verlag.

[LV16] Lebed, V., Vendramin, L. Cohomology and extensions of braces, arXiv preprint arXiv:1601.01633 [math.GR], 2016

[LYZ] Lu, J., Yan, M., and Zhu, Y., On the set-theoretical Yang-Baxter equation, Duke Math. J. 104 (2000), 1-18.

[Ma90] Majid, S., Matched pairs of Lie groups associated to solutions of the Yang-Baxter equations, Pac. J. Math. 141 (1990), 311-332.

[Ma90a] Majid, S., Physics for algebraists: Non-commutative and non-cocommutative Hopf algebras by a bicrosspoduct construction, J. Algebra, 130 (1990) 17-64.

[Ma90b] Majid, S., More examples of bicrossproduct and double cross product Hopf algebras, Israel J. Math, 72 (1990), 133-148.

[Ma95] Majid, S., Foundations of quantum group theory, Cambridge Univ. Press (1995).

[Man] Manin, Yu., Quantum Groups and Non Commutative Geometry Montreal University Report No. CRM- 1561, 1988.

[Mat] Matsumoto D.K., Dynamical braces and dynamical Yang-Baxter maps, J. Pure Appl. Algebra 217 (2013), 195-206.

[MS] Matsumoto D.K., and Shibukawa, Y, Quantum Yang-Baxter equation, braided semigroups, and dynamical Yang-Baxter maps, Tokyo J. of Math. 38, (2015), 227-237.

[Ru05] Rump, W., A decomposition theorem for square-free unitary solutions of the quantum Yang-Baxter equation, Adv. Math. 193 (2005), 40-55.

[Ru06] Rump, W., Modules over braces, Algebra Discr. Math. 2 (2006), 127-137

[Ru07] Rump,W., Braces, radical rings, and the quantum Yang-Baxter equation, J. Algebra 307 (2007), 153-170

[Ru14] Rump, W. The brace of a classical group, Note Mat. 34 (2014), 115-144.

[Smok] Smoktunowicz, A., On Engel groups, nilpotent groups, rings, braces and the Yang-Baxter equation, arXiv:1509.00420, Sept. 2015

[Ta81] Takeuchi, M., Matched pairs of groups and bismash products of Hopf algebras, Commun. Alg. 9 (1981), 841.

[Ta] Takeuchi, M., Survey on matched pairs of groups. An elementary approach to the ESS-LYZ theory, Banach Center Publ. 61 (2003), 305-331.

[Ve] Vendramin, L., Extensions of set-theoretic solutions of the Yang-Baxter equation and a conjecture of Gateva-Ivanova, J. Pure Appl. Algebra, 220 (2016) 2064-2076 .

[WX] Weinstein, A. and Xu, P., Classical solutions of the quantum Yang-Baxter equation, Comm. Math. Phys. 148 (1992), 309-343.

Institute of Mathematics and Informatics, Bulgarian Academy of Sciences, Sofia 1113, Bulgaria, and American University in Bulgaria, Blagoevgrad 2700, Bulgaria

E-mail address: tatyana@aubg.bg, tatianagateva@gmail.com 